Pacific

Journal of

Mathematics

UNFAITHFUL COMPLEX HYPERBOLIC TRIANGLE GROUPS, II: HIGHER ORDER REFLECTIONS

JOHN R. PARKER AND JULIEN PAUPERT 


\title{
UNFAITHFUL COMPLEX HYPERBOLIC TRIANGLE GROUPS, II: HIGHER ORDER REFLECTIONS
}

\author{
JOHN R. PARKER AND JULIEN PAUPERT
}

\begin{abstract}
We consider symmetric complex hyperbolic triangle groups generated by three complex reflections through angle $2 \pi / p$, with $p \geqslant 3$. We restrict our attention to those groups where certain words are elliptic. Our goal is to find necessary conditions for such a group to be discrete. The main application we have in mind is that such groups are candidates for nonarithmetic lattices in $\mathrm{SU}(2,1)$.
\end{abstract}

\section{Introduction}

Mostow [1980] was the first to construct examples of nonarithmetic complex hyperbolic lattices. These lattices were generated by three complex reflections $R_{1}$, $R_{2}$ and $R_{3}$ with the property that there exists a complex hyperbolic isometry $J$ of order 3 so that $R_{j+1}=J R_{j} J^{-1}$ (here and throughout the paper the indices will be taken mod 3). In Mostow's examples the generators $R_{j}$ have order $p=3,4$ or 5. Subsequently Deligne and Mostow constructed further nonarithmetic lattices as monodromy groups of certain hypergeometric functions [Deligne and Mostow 1993; Mostow 1986]. (The lattices from [Deligne and Mostow 1993] in dimension 2 were known to Picard, who did not consider their arithmetic nature.) These lattices are (commensurable with) groups generated by complex reflections $R_{j}$ with other values of $p$; see [Mostow 1986; Sauter 1990]. Subsequently no new nonarithmetic lattices have been constructed.

The case $p=2$ was treated in [Parker 2008]. The author considered complex involutions $I_{1}, I_{2}$ and $I_{3}$ with the property that there is a $J$ of order 3 so that $I_{j+1}=J I_{j} J^{-1}$. In particular, he used a theorem of Conway and Jones [1976] to classify all such groups where $I_{1} I_{2}$ and $I_{1} I_{2} I_{3}$ have finite order.

Remarkably, when $p \geqslant 3$ finding groups for which $R_{1} R_{2}$ and $R_{1} R_{2} R_{3}$ have finite order involves solving the same equation as in the case $p=2$. In this paper we use the solutions to this equation found using [Conway and Jones 1976] in [Parker 2008] in the general case.

MSC2000: 20H10, 22E40, 51M10.

Keywords: complex reflection, complex hyperbolic, lattices in $\mathrm{SU}(2,1)$, nonarithmetic lattice. 
We describe the configuration space of all groups generated by a complex reflection $R_{1}$ of order $p$ and a regular elliptic motion $J$ of order 3. This configuration space is parametrised by the conjugacy class of the product $R_{1} J$, which we represent geometrically in two different manners. The first, following Goldman and Parker, is to consider the trace of $R_{1} J$; this determines the conjugacy class of $R_{1} J$ when it is loxodromic, but there is a threefold indetermination when it is elliptic or parabolic. The second manner, following Paupert, is to use the geometric invariants of the conjugacy class, i.e. an angle pair for elliptic isometries and a pair (angle, length) for loxodromic isometries. We will use both parameter spaces in this paper, where we focus on the elliptic case.

Our first result is the direct analogue of the main theorem of [Parker 2008], and can be roughly stated as follows:

Theorem 1.1. Let $R_{1}$ be a complex reflection of order $p$ and $J$ a regular elliptic isometry of order 3 in $\mathrm{PU}(2,1)$. Suppose that $R_{1} J$ and $R_{1} R_{2}=R_{1} J R_{1} J^{-1}$ are elliptic. If the group $\Gamma=\left\langle R_{1}, J\right\rangle$ is discrete then one of the following is true:

- $\Gamma$ is one of Mostow's lattices.

- $\Gamma$ is a subgroup of one of Mostow's lattices.

- $\Gamma$ is one of the sporadic groups described below.

The sporadic groups correspond to the 18 exceptional solutions from [Conway and Jones 1976], which do not depend on $p$ (the groups do change with $p$ of course). We determine for each $p \geqslant 3$ which of these points lie inside our configuration space. One must then analyse each of these groups to decide whether or not it is discrete, if so whether or not it is a lattice and if so whether or not it is arithmetic. We illustrate ways to attack this problem by showing that certain solutions are arithmetic and certain other solutions are nondiscrete. We analyse in detail the situation for $p=3$, which can be summarised as follows:

Theorem 1.2. There are 16 sporadic groups for $p=3$, with the following properties:

- Four of them fix a point in $\mathbf{H}_{\mathbb{C}}^{2}$.

- One stabilises a complex line.

- One is contained in an arithmetic lattice in $\mathrm{SU}(2,1)$.

- The ten remaining groups are none of the above.

The crucial question is then to determine whether or not the ten remaining groups are discrete. We give a negative answer for four of them, by finding elliptic elements of infinite order in the group. For more details, see Theorem 6.1. 


\section{Parameters, traces and angles}

In this section we show how to use a single complex number $\tau$ to parametrise symmetric complex hyperbolic triangle groups generated by three complex reflections through angle $\psi$. This generalises the construction in [Parker 2008] where involutions (that is $\psi=\pi$ ) were considered.

We give an alternative description following [Paupert 2007], where the same space is parametrised by a pair of angles. It is fairly easy to pass between the two parametrisations and we shall use the one best adapted to each particular problem.

We then describe how the properties of the group (for example the type of $R_{1} R_{2}$ and $R_{1} R_{2} R_{3}$ ) vary with $\tau$. Much of this section follows closely the relevant parts of [Parker 2008]. Otherwise we will try to keep this account as self contained as possible. However, we shall assume a certain amount of background knowledge of complex hyperbolic geometry. For such background material on complex hyperbolic space see [Goldman 1999] and for material on complex hyperbolic triangle groups see [Schwartz 2002] or [Pratoussevitch 2005]. Many of the ideas we use may be traced back to [Mostow 1980].

2.1. Complex reflections through angle $\psi$. Let $L_{1}$ be a complex line in complex hyperbolic 2-space $\mathbf{H}_{\mathbb{C}}^{2}$ and write $R_{1}$ for the complex reflection through angle $\psi$ fixing $L_{1}$. In our applications we shall only consider the case where $\psi=2 \pi / p$ for an integer $p$ at least 3. Points of $L_{1}$ are fixed by $R_{1}$ and $\mathbf{n}_{1}$, the polar vector of $L_{1}$, is sent by $R_{1}$ to $e^{i \psi} \mathbf{n}_{1}$. Hence the matrix in $\mathrm{U}(2,1)$ associated to $R_{1}$ has determinant $e^{i \psi}$. Since we shall be dealing with traces, we want to lift $R_{1}$ to a matrix in $\mathrm{SU}(2,1)$. Hence we multiply the $\mathrm{U}(2,1)$ matrix by $e^{-i \psi / 3}$, and take $R_{1}$ to be the map given by

$$
R_{1}(\mathbf{z})=e^{-i \psi / 3} \mathbf{z}+\left(e^{2 i \psi / 3}-e^{-i \psi / 3}\right) \frac{\left\langle\mathbf{z}, \mathbf{n}_{1}\right\rangle}{\left\langle\mathbf{n}_{1}, \mathbf{n}_{1}\right\rangle} \mathbf{n}_{1} .
$$

We remark that we can add arbitrary multiples of $2 \pi$ to the angle $\psi$. Equivalently, since $\mathrm{SU}(2,1)$ is a triple cover of $\mathrm{PU}(2,1)$ we could have multiplied $R_{1}$ by any power of $e^{2 \pi i / 3}$. Thus in the case where $R_{1}$ has order 2 , in the analogous formula (2-1) in [Parker 2008], the angle $\psi$ was chosen to be $3 \pi$ and so $e^{-i \psi / 3}$ and $e^{2 i \psi / 3}$ become -1 and +1 respectively. Another way to have achieved this would have been to put $\psi=\pi$ in (2-1) and then multiplied this expression by $e^{-2 \pi i / 3}$.

2.2. Traces. We define $\tau=\operatorname{tr}\left(R_{1} J\right)$ to be the parameter in our space. We now show how to express $\tau$ in terms of the polar vectors and Hermitian form. We go on to find the trace of $R_{1} R_{2}$ in the same way.

Our first lemma and its corollary generalise Lemma 2.4 and Corollary 2.5 of [Parker 2008] and are proven in a similar manner. 
Lemma 2.1. Let $A$ be any element of $\mathrm{SU}(2,1)$. Then

$$
\operatorname{tr}\left(R_{1} A\right)=e^{-i \psi / 3} \operatorname{tr}(A)+\left(e^{2 i \psi / 3}-e^{-i \psi / 3}\right) \frac{\left\langle A\left(\mathbf{n}_{1}\right), \mathbf{n}_{1}\right\rangle}{\left\langle\mathbf{n}_{1}, \mathbf{n}_{1}\right\rangle} .
$$

Corollary 2.2. Let $R_{1}$ be a complex reflection through angle $\psi$, fixing a complex line $L_{1}$ with polar vector $\mathbf{n}_{1}$. Let $J \in \mathrm{SU}(2,1)$ be a regular elliptic isometry of order 3. Then

$$
\begin{aligned}
\tau:=\operatorname{tr}\left(R_{1} J\right) & =\left(e^{2 i \psi / 3}-e^{-i \psi / 3}\right) \frac{\left\langle J\left(\mathbf{n}_{1}\right), \mathbf{n}_{1}\right\rangle}{\left\langle\mathbf{n}_{1}, \mathbf{n}_{1}\right\rangle} \\
& =2 \sin (\psi / 2) i e^{i \psi / 6} \frac{\left\langle J\left(\mathbf{n}_{1}\right), \mathbf{n}_{1}\right\rangle}{\left\langle\mathbf{n}_{1}, \mathbf{n}_{1}\right\rangle} .
\end{aligned}
$$

Proposition 2.3 ([Goldman 1999, Theorem 6.2.4]). Let $\tau$ be given by (2-2). Then $R_{1} J$ is regular elliptic if and only if $\tau$ lies in the region

$$
\boldsymbol{\Delta}=\left\{z \in \mathbb{C}:|z|^{4}-8 \operatorname{Re} z^{3}+18|z|^{2}-27<0\right\} .
$$

The curve bounding the region $\boldsymbol{\Delta}$ of Proposition 2.3 is a deltoid. Groups for which $R_{1} J$ is regular elliptic correspond to points in $\Delta$. Points on the boundary deltoid correspond to points where $R_{1} J$ has repeated eigenvalues, and so is either a complex reflection or is parabolic. Since $R_{1} R_{2} R_{3}=\left(R_{1} J\right)^{3}$ we can determine the type of $R_{1} R_{2} R_{3}$ from $R_{1} J$. (Note that it may be that $R_{1} J$ is regular elliptic and that $R_{1} R_{2} R_{3}$ is a complex reflection.)

We now consider $R_{1} R_{2}$, and hence by symmetry $R_{2} R_{3}$ and $R_{3} R_{1}$ as well. This generalises Lemma 2.7 of [Parker 2008] and its proof is analogous.

Proposition 2.4. Let $L_{1}$ be a complex line in $\mathbf{H}_{\mathbb{C}}^{2}$ with polar vector $\mathbf{n}_{1}$. Let $J \in$ $\mathrm{SU}(2,1)$ be a regular elliptic isometry of order 3 and write $L_{2}=J\left(L_{1}\right)$. Let $R_{1}$ and $R_{2}=J R_{1} J^{-1}$ denote the complex reflections through angle $\psi$ fixing $L_{1}$ and $L_{2}$ respectively. Then

$$
\operatorname{tr}\left(R_{1} R_{2}\right)=e^{i \psi / 3}\left(2-|\tau|^{2}\right)+e^{-2 i \psi / 3}
$$

where $\tau$ is given by (2-2).

Corollary 2.5. If $|\tau|>2$ then $R_{1} R_{2}$ is loxodromic. If $|\tau|=2 \cos (\theta)$ then $R_{1} R_{2}$ has eigenvalues $e^{-2 i \psi / 3},-e^{i \psi / 3+2 i \theta}$ and $-e^{i \psi / 3-2 i \theta}$.

Proof. Each point on $L_{1}$ is an $e^{-i \psi / 3}$ eigenvector for $R_{1}$ and each point on $L_{2}$ is an $e^{-i \psi / 3}$ eigenvector for $R_{2}$. Therefore if $\mathbf{z} \in \mathbb{C}^{2,1}$ lies on the intersection of $L_{1}$ and $L_{2}$ we have

$$
R_{1} R_{2}(\mathbf{z})=e^{-i \psi / 3} R_{1}(\mathbf{z})=e^{-2 i \psi / 3} \mathbf{z} .
$$

Hence $\mathbf{z}$ is an $e^{-2 i \psi / 3}$ eigenvector for $R_{1} R_{2}$. 
If $|\tau|>2$ then $R_{1} R_{2}$ has an eigenvalue whose modulus is greater than one. Hence $R_{1} R_{2}$ is loxodromic.

So we assume that $|\tau|=2 \cos (\theta)$. Since $R_{1} R_{2}$ has an eigenvalue $e^{-2 i \psi / 3}$ and determinant 1 , we see that its other eigenvalues must be $-e^{i \psi / 3+2 i \theta}$ and $-e^{i \psi / 3-2 i \theta}$ as claimed.

2.3. Angles. Conjugacy classes of elliptic isometries in $\mathrm{PU}(2,1)$ are characterised by an unordered angle pair $\left\{\theta_{1}, \theta_{2}\right\}$ with $\theta_{i} \in \mathbb{R} / 2 \pi \mathbb{Z}$. These angles can be defined by noting that an elliptic isometry $g$ belongs to a maximal compact subgroup of $\mathrm{PU}(2,1)$, which is a group conjugate to $\mathrm{U}(2)$. Then all elements of $\mathrm{U}(2)$ which are conjugate to $g$ have the same eigenvalues of norm 1, which we define to be $e^{i \theta_{1}}$ and $e^{i \theta_{2}}$. In concrete terms, a matrix $A \in \mathrm{U}(2,1)$ whose associated isometry $g \in \mathrm{PU}(2,1)$ is elliptic is semisimple with eigenvalues of norm 1 , say $e^{i \alpha_{1}}, e^{i \alpha_{2}}$ and $e^{i \alpha_{3}}$. One of these is of negative type in the sense that the associated eigenspace intersects the negative cone in $\mathbb{C}^{3}$ of the ambient Hermitian form (of signature $(2,1))$. Supposing for instance that $e^{i \alpha_{3}}$ is of negative type, the angle pair of $g$ is then $\left\{\alpha_{1}-\alpha_{3}, \alpha_{2}-\alpha_{3}\right\}$. It is thus in general not sufficient to know the eigenvalues of an elliptic matrix to obtain the angle pair of the corresponding isometry; one must determine which of them is of negative type.

This can also be seen in terms of the trace of matrix representatives in $\mathrm{SU}(2,1)$. Recall from [Goldman 1999] that elliptic isometries have matrix representatives in $\mathrm{SU}(2,1)$ whose trace lies in the closure of the region $\boldsymbol{\Delta}$ given in (2-3). Multiplication of $A$, and hence of its trace, by a cube root of unity corresponds to the same complex hyperbolic isometry in $\mathrm{PU}(2,1)$. Up to this ambiguity, the map from angle pairs to traces is given by

$$
\left\{\theta_{1}, \theta_{2}\right\} \mapsto e^{2 i \theta_{1} / 3-i \theta_{2} / 3}+e^{2 i \theta_{2} / 3-i \theta_{1} / 3}+e^{-i \theta_{1} / 3-i \theta_{2} / 3} .
$$

This map is three-to-one, except at the exceptional central point $\{4 \pi / 3,2 \pi / 3\}$ which is the only preimage of 0 . The three preimages in this case correspond to the fact that one of the three eigenvalues has negative type. There are three possible choices and the trace is the same for each of them. Conversely, given a trace $\tau=e^{i \alpha}+e^{i \beta}+e^{-i \alpha-i \beta}$ the three preimages of this trace are the three angle pairs

$$
\{2 \alpha+\beta, \alpha+2 \beta\}, \quad\{\alpha-\beta,-\alpha-2 \beta\}, \quad\{\beta-\alpha,-2 \alpha-\beta\} .
$$

In order to get these into the parameter space where $0 \leqslant \theta_{1} \leqslant 2 \pi$ and $0 \leqslant \theta_{2} \leqslant \theta_{1}$ one may have to add a multiple of $2 \pi$ to either or both angles and one may have to change their order. See [Paupert 2005, pp. 29-30] for more details on angle pairs and their relation to traces. 
2.4. The trace parameter space. Suppose we are given a symmetric configuration of three complex lines $L_{1}, L_{2}$ and $L_{3}$ with polar vectors $\mathbf{n}_{1}, \mathbf{n}_{2}$ and $\mathbf{n}_{3}$. This means that there exists a regular elliptic isometry $J$ of order 3 such that $J\left(L_{j}\right)=$ $L_{j+1}$ (with $j=1,2,3$ taken mod 3), or equivalently $J\left(\mathbf{n}_{j}\right)=\mathbf{n}_{j+1}$ (where $J$ is understood to act on all of $\mathbb{C} P^{2}$ ). Because $J$ preserves the Hermitian form, $\left\langle\mathbf{n}_{j}, \mathbf{n}_{j}\right\rangle$ is the same positive real number for each $j$. We normalise $\mathbf{n}_{j}$ so that this number is $2 \sin (\psi / 2)$. Likewise $\left\langle\mathbf{n}_{j+1}, \mathbf{n}_{j}\right\rangle=\left\langle J\left(\mathbf{n}_{j}\right), \mathbf{n}_{j}\right\rangle$ is the same complex number for each $j$. Using Corollary 2.2 we see that

$$
\left\langle J\left(\mathbf{n}_{j}\right), \mathbf{n}_{j}\right\rangle=\frac{\tau\left\langle\mathbf{n}_{j}, \mathbf{n}_{j}\right\rangle}{e^{2 i \psi / 3}-e^{-i \psi / 3}}=-i e^{-i \psi / 6} \tau .
$$

That is,

$$
\begin{aligned}
& \left\langle\mathbf{n}_{1}, \mathbf{n}_{1}\right\rangle=\left\langle\mathbf{n}_{2}, \mathbf{n}_{2}\right\rangle=\left\langle\mathbf{n}_{3}, \mathbf{n}_{3}\right\rangle=2 \sin (\psi / 2), \\
& \left\langle\mathbf{n}_{2}, \mathbf{n}_{1}\right\rangle=\left\langle\mathbf{n}_{3}, \mathbf{n}_{2}\right\rangle=\left\langle\mathbf{n}_{1}, \mathbf{n}_{3}\right\rangle=-i e^{-i \psi / 6} \tau .
\end{aligned}
$$

All of this works for any Hermitian form of signature $(2,1)$. We now make a choice of vectors $\mathbf{n}_{1}, \mathbf{n}_{2}$ and $\mathbf{n}_{3}$. This determines a Hermitian form. We choose

$$
\mathbf{n}_{1}=\left[\begin{array}{l}
1 \\
0 \\
0
\end{array}\right], \quad \mathbf{n}_{2}=\left[\begin{array}{l}
0 \\
1 \\
0
\end{array}\right], \quad \mathbf{n}_{3}=\left[\begin{array}{l}
0 \\
0 \\
1
\end{array}\right] .
$$

Together with (2-2), this means that, with this choice, the Hermitian form must be $\langle\mathbf{z}, \mathbf{w}\rangle=\mathbf{w}^{*} H_{\tau} \mathbf{z}$, where

$$
H_{\tau}=\left[\begin{array}{ccc}
2 \sin (\psi / 2) & -i e^{-i \psi / 6} \tau & i e^{i \psi / 6} \bar{\tau} \\
i e^{i \psi / 6} \bar{\tau} & 2 \sin (\psi / 2) & -i e^{-i \psi / 6} \tau \\
-i e^{-i \psi / 6} \tau & i e^{i \psi / 6} \bar{\tau} & 2 \sin (\psi / 2)
\end{array}\right] .
$$

We can immediately write down

$$
J=\left[\begin{array}{lll}
0 & 0 & 1 \\
1 & 0 & 0 \\
0 & 1 & 0
\end{array}\right]
$$

and, using (2-1), the expressions for the reflections $R_{j}$ :

$$
\begin{aligned}
& R_{1}=\left[\begin{array}{ccc}
e^{2 i \psi / 3} & \tau & -e^{i \psi / 3} \bar{\tau} \\
0 & e^{-i \psi / 3} & 0 \\
0 & 0 & e^{-i \psi / 3}
\end{array}\right], \quad R_{2}=\left[\begin{array}{ccc}
e^{-i \psi / 3} & 0 & 0 \\
-e^{i \psi / 3} \bar{\tau} & e^{2 i \psi / 3} & \tau \\
0 & 0 & e^{-i \psi / 3}
\end{array}\right] \\
& R_{3}=\left[\begin{array}{ccc}
e^{-i \psi / 3} & 0 & 0 \\
0 & e^{-i \psi / 3} & 0 \\
\tau & -e^{i \psi / 3} \bar{\tau} & e^{2 i \psi / 3}
\end{array}\right] \text {. }
\end{aligned}
$$


From this it is clear that the groups $\Gamma=\left\langle R_{1}, J\right\rangle$ and $\Delta=\left\langle R_{1}, R_{2}, R_{3}\right\rangle$ are completely determined up to conjugacy by the parameter $\tau$. However, not all values of $\tau$ correspond to complex hyperbolic triangle groups. It may be that the Hermitian matrix $H_{\tau}$ does not have signature $(2,1)$. We now determine this by finding the eigenvalues of $H_{\tau}$. In this lemma and throughout the paper we write $\omega=e^{2 \pi i / 3}=(-1+i \sqrt{3}) / 2$.

In what follows we will be interested in the case where $R_{1} J$ is elliptic. In this case its eigenvalues are unit modulus complex numbers whose product is 1 . We write them as $e^{i \alpha}, e^{i \beta}$ and $e^{-i \alpha-i \beta}$. This means that $\tau=e^{i \alpha}+e^{i \beta}+e^{-i \alpha-i \beta}$.

Lemma 2.6. Let $H_{\tau}$ be given by (2-5) where $\tau=e^{i \alpha}+e^{i \beta}+e^{-i \alpha-i \beta}$. Then the eigenvalues of $H_{\tau}$ are

$-8 \sin (\alpha / 2+\psi / 6+2 k \pi / 3) \sin (\beta / 2+\psi / 6+2 k \pi / 3) \sin (-\alpha / 2-\beta / 2+\psi / 6+2 k \pi / 3)$

for $k=0,1,2$.

Proof. We could solve the characteristic polynomial of $H_{\tau}$. However, it is easier to observe that eigenvectors for $H_{\tau}$ are

$$
\left[\begin{array}{l}
1 \\
1 \\
1
\end{array}\right], \quad\left[\begin{array}{l}
1 \\
\bar{\omega} \\
\omega
\end{array}\right], \quad\left[\begin{array}{c}
1 \\
\omega \\
\bar{\omega}
\end{array}\right] .
$$

We can immediately write down the eigenvalues as

$$
\lambda_{k}=2 \sin (\psi / 2)-i e^{-i \psi / 6-2 k \pi i / 3} \tau+i e^{i \psi / 6+2 k \pi i / 3} \bar{\tau}
$$

for $k=0,1,2$. Subsitituting for $\tau$ gives

$$
\begin{aligned}
\lambda_{k}=2 \sin (\psi / 2)+2 \sin (-\alpha & -\beta-\psi / 6-2 k \pi / 3) \\
& +2 \sin (\alpha-\psi / 6-2 k \pi / 3)+2 \sin (\beta-\psi / 6-2 k \pi / 3) .
\end{aligned}
$$

Using elementary trigonometry we see that this equals $-8 \sin (\alpha / 2+\psi / 6+2 k \pi / 3) \sin (\beta / 2+\psi / 6+2 k \pi / 3) \sin (-\alpha / 2-\beta / 2+\psi / 6+2 k \pi / 3)$.

Corollary 2.7. When $\tau=e^{i \alpha}+e^{i \beta}+e^{-i \alpha-i \beta}$ the matrix $H_{\tau}$ has signature $(2,1)$ if and only if

$$
8 \sin (3 \alpha / 2+\psi / 2) \sin (3 \beta / 2+\psi / 2) \sin (-3(\alpha+\beta) / 2+\psi / 2)<0 .
$$

Proof. $H_{\tau}$ has positive trace, so its signature is $(2,1)$ if and only if its determinant is negative. Using the identity $\sin (3 \theta)=-4 \sin (\theta) \sin (\theta+2 \pi / 3) \sin (\theta+4 \pi / 3)$ 
we see that the product of the three eigenvalues is

$$
8 \sin (3 \alpha / 2+\psi / 2) \sin (3 \beta / 2+\psi / 2) \sin (-3(\alpha+\beta) / 2+\psi / 2) .
$$

Hence $H_{\tau}$ has signature $(2,1)$ if and only if this expression is negative.

The locus where each eigenvalue is zero corresponds to a line in $\mathbb{C}$. These lines divide $\mathbb{C}$ into seven components which fall into three types:

- The central triangle. Here all three eigenvalues are positive and $H_{\tau}$ is positive definite.

- Three infinite components that each share a common edge with the central triangle. Here two eigenvalues are positive and one negative. Hence $H_{\tau}$ has signature $(2,1)$. This is our parameter space.

- Three infinite components that each only abut the central triangle in a point. Here one eigenvalue is positive and two are negative. These correspond to groups of complex hyperbolic isometries generated by three complex reflections that each fix a point.

Therefore the parameter space we are interested in, that is those values of $\tau$ satisfying (2-7), has three components related by multiplication by powers of $\omega=e^{2 \pi i / 3}$. This corresponds to the fact that $J \in \mathrm{SU}(2,1)$ is only defined up to multiplication by a cube root of unity. Hence $\tau$ is only defined up to a cube root of unity. If we factor out by this equivalence, our parameter space becomes one of these components.

2.5. Arithmetic. When we are discussing the discreteness of $\left\langle R_{1}, R_{2}, R_{3}\right\rangle$ below, we shall analyse whether or not the group is arithmetic. In order to do so one must find a suitable ring containing the matrix entries (after possibly conjugating).

Proposition 2.8. The maps $R_{1}, R_{2}$ and $R_{3}$ may be conjugated within $\mathrm{SU}(2,1)$ and scaled so that their matrix entries lie in the ring $\mathbb{Z}\left[\tau, \bar{\tau}, e^{i \psi}, e^{-i \psi}\right]$.

Proof. Conjugating the matrices $R_{1}, R_{2}$ and $R_{3}$ above by $C=\operatorname{diag}\left(e^{-i \psi / 3}, 1, e^{i \psi / 3}\right)$ gives

$$
\begin{gathered}
C R_{1} C^{-1}=e^{-i \psi / 3}\left[\begin{array}{ccc}
e^{i \psi} & \tau & -\bar{\tau} \\
0 & 1 & 0 \\
0 & 0 & 1
\end{array}\right], \quad C R_{2} C^{-1}=e^{-i \psi / 3}\left[\begin{array}{ccc}
1 & 0 & 0 \\
-e^{i \psi} \bar{\tau} & e^{i \psi} & \tau \\
0 & 0 & 1
\end{array}\right], \\
C R_{3} C^{-1}=e^{-i \psi / 3}\left[\begin{array}{ccc}
1 & 0 & 0 \\
0 & 1 & 0 \\
e^{i \psi} \tau & -e^{i \psi} \bar{\tau} & e^{i \psi}
\end{array}\right]
\end{gathered}
$$

Hence the group generated by $e^{i \psi / 3} C R_{j} C^{-1}$ for $j=1,2,3$ consists of matrices whose entries lie in the ring $\mathbb{Z}\left[\tau, \bar{\tau}, e^{i \psi}, e^{-i \psi}\right]$. 
It is very important to keep track of what happens to $H_{\tau}$ when performing this conjugation. The matrices above preserve the Hermitian form given by (any real multiple of) $\mathrm{CH}_{\tau} \mathrm{C}^{-1}$. So we may take the Hermitian form to be given by (2-8)

$$
2 \sin (\psi / 2) C H_{\tau} C^{-1}=\left[\begin{array}{ccc}
2-e^{i \psi}-e^{-i \psi} & \left(e^{-i \psi}-1\right) \tau & \left(1-e^{-i \psi}\right) \bar{\tau} \\
\left(e^{i \psi}-1\right) \bar{\tau} & 2-e^{i \psi}-e^{-i \psi} & \left(e^{-i \psi}-1\right) \tau \\
\left(1-e^{i \psi}\right) \tau & \left(e^{i \psi}-1\right) \bar{\tau} & 2-e^{i \psi}-e^{-i \psi}
\end{array}\right] .
$$

Hence the entries of $2 \sin (\psi / 2) C H_{\tau} C^{-1}$ also lie in the $\operatorname{ring} \mathbb{Z}\left[\tau, \bar{\tau}, e^{i \psi}, e^{-i \psi}\right]$.

2.6. The angle parameter space. Paupert [2007] characterised which angle pairs can arise for the product $A B$ (when it is elliptic), as $A$ and $B$ each vary inside a fixed elliptic conjugacy class (i.e., each have a fixed angle pair). In the present case, $R_{1}$ (the first generator) has angles $\{0,2 \pi / p\}$, and $J$ (the second generator) has angles $\{4 \pi / 3,2 \pi / 3\}$. The allowable region in the surface $\mathbb{T}^{2} / \mathfrak{S}_{2}$ is then a convex pentagon (degenerating to a triangle for $p=3$ ) with the following properties, which we quote from [Paupert 2007]. The two "totally reducible vertices" $V_{1}$ and $V_{2}$ (points representing an abelian group) have respective coordinates $\{4 \pi / 3,2 \pi / 3+2 \pi / p\}$ and $\{4 \pi / 3+2 \pi / p, 2 \pi / 3\}$. These two vertices are joined by a line segment of slope -1 corresponding to the reducible groups which fix a point inside $\mathbf{H}_{\mathbb{C}}^{2}$. Each of these vertices is also the endpoint of a line segment corresponding to the reducible groups which stabilise a complex line. The first of these segments starts upward at $V_{1}$ with slope 2 , bounces off the diagonal and goes on (with slope $1 / 2$ ) until it reaches the boundary of the square. The second segment starts upward at $V_{2}$ with slope $1 / 2$ and goes off to the boundary. The last side of the quadrilateral is the resulting portion of the boundary segment $\{2 \pi\} \times[0,2 \pi]$; in all corresponding groups the product $R_{1} J$ is parabolic, as was proven in [Paupert 2007]. The polygons for $p=3, \ldots, 10$ are illustrated in Figure 1. In what follows we shall assume that $R_{1} R_{2}$ is elliptic. From Corollary 2.5, we see that this implies $|\tau| \leq 2$. This condition does not have a striaghtforward interpretation in the angle coordinates. In Figure 1 we have drawn the curve corresponding to $|\tau|=2$.

\section{When $R_{1} R_{2} R_{3}$ is elliptic and $R_{1} R_{2}$ is nonloxodromic}

We restrict our attention to those groups for which $R_{1} R_{2} R_{3}$ is elliptic of finite order and $R_{1} R_{2}$ is either elliptic of finite order or parabolic. These are groups for which $\tau$ lies inside or on the deltoid and inside or on the circle $|\tau|=2$. Since they have finite order, the eigenvalues of $R_{1} R_{2} R_{3}$ and $R_{1} R_{2}$ are all roots of unity. This fact leads to a linear equation in certain cosines of rational multiples of $\pi$. We find all solutions to this equation using a theorem of Conway and Jones [1976]. We then go on to find which of these solutions lie in our parameter space. 
3.1. The eigenvalue equation. We now investigate when both $R_{1} R_{2}$ and $R_{1} R_{2} R_{3}$ are elliptic of finite order. (Our proof will be valid when $R_{1} R_{2}$ is parabolic as well.) We know that $R_{1} J$ (and hence $R_{1} R_{2} R_{3}$ ) is elliptic of finite order if and only if

$$
\tau=\operatorname{tr}\left(R_{1} J\right)=e^{i \alpha}+e^{i \beta}+e^{-i \alpha-i \beta},
$$

where $\alpha$ and $\beta$ are rational multiples of $\pi$. Likewise for $R_{1} R_{2}$. In fact we know slightly more. Since the intersection of $L_{1}$ and $L_{2}$ is an $e^{-i \psi / 3}$-eigenvector for each of $R_{1}$ and $R_{2}$ it must be a $e^{-2 i \psi / 3}$-eigenvector for $R_{1} R_{2}$. From Proposition 2.4 we know that

$$
\operatorname{tr}\left(R_{1} R_{2}\right)=e^{i \psi / 3}\left(2-|\tau|^{2}\right)+e^{-2 i \psi / 3} .
$$

Hence the other two eigenvalues of $R_{1} R_{2}$ are $-e^{i \psi / 3+2 i \theta}$ and $-e^{i \psi / 3-2 i \theta}$. (We have taken minus signs in order to make our angles agree with [Parker 2008].) Thus

$$
|\tau|^{2}-2=2 \cos (2 \theta)
$$

where $\theta$ is a rational multiple of $\pi$.

We solve equations (3-1) and (3-2) by eliminating $\tau$. That is, we seek $\theta, \alpha, \beta$ rational multiples of $\pi$ so that

$$
2 \cos (2 \theta)+2=|\tau|^{2}=3+2 \cos (\alpha-\beta)+2 \cos (\alpha+2 \beta)+2 \cos (-2 \alpha-\beta) .
$$

Rearranging, this becomes

$$
\frac{1}{2}=\cos (2 \theta)-\cos (\alpha-\beta)-\cos (\alpha+2 \beta)-\cos (-2 \alpha-\beta) .
$$

Parker [2008] used a theorem of Conway and Jones [1976] to solve (3-3). Up to complex conjugating $\tau$ (so changing the sign of $\alpha$ and $\beta$ ) and multiplying by a power of $\omega$, the only solutions are those indicated in the table:

\begin{tabular}{|l|l|lll|lll|}
\hline & $2 \theta$ & $\alpha-\beta$ & $\alpha+2 \beta$ & $2 \alpha+\beta$ & $\alpha$ & $\beta$ & $\alpha+\beta$ \\
\hline (i) & $2 \pi / 3$ & $\pi-\phi / 2$ & $\pi$ & $2 \pi-\phi / 2$ & $\pi-\phi / 3$ & $\phi / 6$ & $\pi-\phi / 6$ \\
(ii) & $\phi$ & $\pi / 3-\phi$ & $\pi / 3+\phi$ & $2 \pi / 3$ & $\pi / 3-\phi / 3$ & $2 \phi / 3$ & $\pi / 3+\phi / 3$ \\
(iii) & $\pi / 3$ & $\pi / 4$ & $\pi / 2$ & $3 \pi / 4$ & $\pi / 3$ & $\pi / 12$ & $5 \pi / 12$ \\
(iv) & $\pi / 5$ & $3 \pi / 10$ & $2 \pi / 5$ & $7 \pi / 10$ & $\pi / 3$ & $\pi / 30$ & $11 \pi / 30$ \\
(v) & $3 \pi / 5$ & $\pi / 10$ & $4 \pi / 5$ & $9 \pi / 10$ & $\pi / 3$ & $7 \pi / 30$ & $17 \pi / 30$ \\
(vi) & $\pi / 2$ & $2 \pi / 7$ & $4 \pi / 7$ & $6 \pi / 7$ & $8 \pi / 21$ & $2 \pi / 21$ & $10 \pi / 21$ \\
(vii) & $\pi / 2$ & $\pi / 15$ & $11 \pi / 15$ & $4 \pi / 5$ & $13 \pi / 45$ & $2 \pi / 9$ & $23 \pi / 45$ \\
(viii) & $\pi / 2$ & $7 \pi / 15$ & $17 \pi / 15$ & $8 \pi / 5$ & $31 \pi / 45$ & $2 \pi / 9$ & $41 \pi / 45$ \\
(ix) & $\pi / 7$ & $\pi / 21$ & $4 \pi / 7$ & $13 \pi / 21$ & $2 \pi / 9$ & $11 \pi / 63$ & $25 \pi / 63$ \\
(x) & $5 \pi / 7$ & $5 \pi / 21$ & $19 \pi / 21$ & $8 \pi / 7$ & $29 \pi / 63$ & $2 \pi / 9$ & $43 \pi / 63$ \\
(xi) & $3 \pi / 7$ & $11 \pi / 21$ & $25 \pi / 21$ & $12 \pi / 7$ & $47 \pi / 63$ & $2 \pi / 9$ & $61 \pi / 63$ \\
\hline
\end{tabular}


We then write down $\tau=\operatorname{tr}\left(R_{1} J\right)=e^{i \alpha}+e^{i \beta}+e^{-i \alpha-i \beta}$ and $\operatorname{tr}\left(R_{1} R_{2} R_{3}\right)=$ $e^{3 i \alpha}+e^{3 i \beta}+e^{-3 i \alpha-3 i \beta}$ using this table. As indicated earlier, the parameters $\tau \omega^{j}$ for $j=1,2$ correspond to the same group as $\tau$.

Proposition 3.1 [Parker 2008, Proposition 3.2]. Suppose that $R_{1} R_{2}$ and $R_{1} R_{2} R_{3}$ are both elliptic of finite order (or possibly $R_{1} R_{2}$ is parabolic). Up to complex conjugating $\tau$ and multiplying by a power of $\omega$, then one of the following is true:

(i) $\tau=-e^{-i \phi / 3}=: \mathrm{m}(\phi)$ for some angle $\phi$;

(ii) $\tau=e^{2 i \phi / 3}+e^{-i \phi / 3}=e^{i \phi / 6} 2 \cos (\phi / 2)=: \mathrm{s}(\phi)$, for some angle $\phi$;

(iii) $\tau=e^{i \pi / 3}+e^{-i \pi / 6} 2 \cos (\pi / 4)=: \sigma(\pi / 6, \pi / 4)$;

(iv) $\tau=e^{i \pi / 3}+e^{-i \pi / 6} 2 \cos (\pi / 5)=: \sigma(\pi / 6, \pi / 5)$;

(v) $\tau=e^{i \pi / 3}+e^{-i \pi / 6} 2 \cos (2 \pi / 5)=: \sigma(\pi / 6,2 \pi / 5)$;

(vi) $\tau=e^{2 \pi i / 7}+e^{4 \pi i / 7}+e^{8 \pi i / 7}=: \sigma(2 \pi / 7)$;

(vii) $\tau=e^{2 \pi i / 9}+e^{-i \pi / 9} 2 \cos (2 \pi / 5)=: \sigma(\pi / 9,2 \pi / 5)$;

(viii) $\tau=e^{2 \pi i / 9}+e^{-\pi i / 9} 2 \cos (4 \pi / 5)=: \sigma(\pi / 9,4 \pi / 5)$;

(ix) $\tau=e^{2 \pi i / 9}+e^{-i \pi / 9} 2 \cos (2 \pi / 7)=: \sigma(\pi / 9,2 \pi / 7)$;

(x) $\tau=e^{2 \pi i / 9}+e^{-i \pi / 9} 2 \cos (4 \pi / 7)=: \sigma(\pi / 9,4 \pi / 7)$;

(xi) $\tau=e^{2 \pi i / 9}+e^{-i \pi / 9} 2 \cos (6 \pi / 7)=: \sigma(\pi / 9,6 \pi / 7)$.

Before proceeding we explain the notation. The possible solutions $\tau$ either lie on one of two curves or are on a finite list of points. The points on the curve from part (i) (for $p \geqslant 3$ ) correspond to Mostow's groups $\Gamma(p, t)$; compare [Mostow 1980]. So we call this the Mostow curve and denote points on it by $\mathrm{m}(\phi)$. The points on the curve from part (ii) correspond to subgroups of Mostow's groups, by the results of Section 5.1. These results generalise the isomorphisms discovered by Sauter [1990], so we call this the Sauter curve and denote points on it by s $(\phi)$. The remaining parts (iii) to (xi) consist of finitely many isolated points which we call sporadic. All the points except for those in part (vi) depend on two angles and so we write them as $\sigma(\phi, \eta)=e^{2 i \phi}+e^{-i \phi} 2 \cos (\eta)$. Note that complex conjugation sends $\sigma(\phi, \eta)$ to $\sigma(-\phi, \eta)$ and multiplication by $e^{2 \pi i / 3}$ sends $\sigma(\phi, \eta)$ to $\sigma(\phi-2 \pi / 3, \eta)$.

We use these various values of $\tau=\operatorname{tr}\left(R_{1} J\right)$ to parametrise groups in $\operatorname{SU}(2,1)$; note that while the values of $\tau$ in the above list do not depend on $\psi$ (the angle of rotation of the reflection $R_{1}$ ), the corresponding groups do vary with $\psi$. We will denote by $\Gamma(\psi, \tau)$ the subgroup of $\mathrm{SU}(2,1)$ generated by $R_{1}$ and $J$, where the rotation angle of $R_{1}$ is $\psi$ and the trace of $R_{1} J$ is $\tau$. Note that there should be no confusion with Mostow's notation $\Gamma(p, t)$, where $p$ is an integer and $t$ is real. Explicitly, by a simple computation using Mostow's generator $R_{1}$ (appropriately normalised in $\mathrm{SU}(2,1))$, Mostow's group $\Gamma(p, t)$ is our $\Gamma\left(2 \pi / p, e^{\pi i(3 / 2+1 /(3 p)-t / 3)}\right)$. 
3.2. The points in angle parameters. We now list the corresponding points in the angle parameter space. This involves considering the differences of the eigenvalues and making sure we have a pair $\left\{\theta_{1}, \theta_{2}\right\}$ with $4 \pi / 3 \leqslant \theta_{1} \leqslant 2 \pi$ and $2 \pi / 3 \leqslant \theta_{2} \leqslant \theta_{1}$.

For the groups of Mostow and Sauter type the corresponding points in the angle space are piecewise linear curves. We now write these down.

Lemma 3.2. (i) The point $\tau=\mathrm{m}(\phi)=-e^{-i \phi / 3}$ corresponds to the angle pair $\{2 \pi-\phi / 2, \pi\}$ if $\phi / 2 \in[0, \pi]$ and $\{2 \pi+\phi / 2, \pi+\phi / 2\}$ if $\phi / 2 \in[-\pi, 0]$.

(ii) The point $\tau=\mathrm{s}(\phi)=e^{2 i \phi / 3}+e^{-i \phi / 3}$ corresponds to the angle pair $\{5 \pi / 3+$ $\phi, 4 \pi / 3\}$ if $\phi \in[-\pi / 3, \pi / 3]$ and $\{7 \pi / 3-\phi, 5 \pi / 3-\phi\}$ if $\phi \in[\pi / 3, \pi]$.

For the sporadic groups, in each case for $\tau$ the angle pair is $\{2 \pi+\beta-\alpha, 2 \pi-$ $2 \alpha-\beta\}$ and for $\bar{\tau}$ it is either $\{2 \pi-\alpha-2 \beta, 2 \pi-2 \alpha-\beta\}$ or $\{2 \pi+\beta-\alpha, \alpha+2 \beta\}$. These angles may be read off from the table on page 366 .

\begin{tabular}{|l|l|l|}
\hline & $\tau$ & $\left\{\theta_{1}, \theta_{2}\right\}$ \\
\hline (iii) & $e^{\pi i / 3}+e^{-\pi i / 6} 2 \cos (\pi / 4)$ & $\{7 \pi / 4,5 \pi / 4\}$ \\
& $e^{-\pi i / 3}+e^{\pi i / 6} 2 \cos (\pi / 4)$ & $\{3 \pi / 2,5 \pi / 4\}$ \\
(iv) & $e^{\pi i / 3}+e^{-\pi i / 6} 2 \cos (\pi / 5)$ & $\{17 \pi / 10,13 \pi / 10\}$ \\
& $e^{-\pi i / 3}+e^{\pi i / 6} 2 \cos (\pi / 5)$ & $\{8 \pi / 5,13 \pi / 10\}$ \\
(v) & $e^{\pi i / 3}+e^{-\pi i / 6} 2 \cos (2 \pi / 5)$ & $\{19 \pi / 10,11 \pi / 10\}$ \\
& $e^{-\pi i / 3}+e^{\pi i / 6} 2 \cos (2 \pi / 5)$ & $\{19 \pi / 10,4 \pi / 5\}$ \\
(vi) & $e^{2 \pi i / 7}+e^{4 \pi i / 7}+e^{8 \pi i / 7}$ & $\{10 \pi / 7,8 \pi / 7\}$ \\
(vii) & $e^{-2 \pi i / 7}+e^{-4 \pi i / 7}+e^{-8 \pi i / 7}$ & $\{12 \pi / 7,8 \pi / 7\}$ \\
(viii) & $e^{2 \pi i / 9}+e^{-\pi i / 9} 2 \cos (2 \pi / 5)$ & $\{29 \pi / 15,18 \pi / 15\}$ \\
& $e^{-2 \pi i / 9}+e^{\pi i / 9} 2 \cos (2 \pi / 5)$ & $\{29 \pi / 15,11 \pi / 15\}$ \\
(ix) & $e^{2 \pi i / 9}+e^{-\pi i / 9} 2 \cos (4 \pi / 5)$ & $\{8 \pi / 5,17 \pi / 15\}$ \\
& $e^{-2 \pi i / 9}+e^{\pi i / 9} 2 \cos (4 \pi / 5)$ & $\{23 \pi / 15,17 \pi / 15\}$ \\
(x) & $e^{2 \pi i / 9}+e^{-\pi i / 9} 2 \cos (2 \pi / 7)$ & $\{41 \pi / 21,29 \pi / 21\}$ \\
& $e^{-2 \pi i / 9}+e^{\pi i / 9} 2 \cos (2 \pi / 7)$ & $\{10 \pi / 7,29 \pi / 21\}$ \\
(xi) & $e^{2 \pi i / 9}+e^{-\pi i / 9} 2 \cos (4 \pi / 7)$ & $\{37 \pi / 21,6 \pi / 7\}$ \\
& $e^{-2 \pi i / 9}+e^{\pi i / 9} 2 \cos (4 \pi / 7)$ & $\{37 \pi / 21,19 \pi / 21\}$ \\
& $e^{2 \pi i / 9}+e^{-\pi i / 9} 2 \cos (6 \pi / 7)$ & $\{12 \pi / 7,25 \pi / 21\}$ \\
& $e^{-2 \pi i / 9}+e^{\pi i / 9} 2 \cos (6 \pi / 7)$ & $\{31 \pi / 21,25 \pi / 21\}$ \\
\hline
\end{tabular}

3.3. Points in parameter space. In this section we consider the values of $\tau$ from Proposition 3.1 or the corresponding angle pairs and we determine which of them is in the parameter space. In each case, we are only interested in the case where $\psi=2 \pi / p$ for some integer $p \geqslant 2$. In each case we know the eigenvalues $e^{i \alpha}$, $e^{i \beta}$ and $e^{-i \alpha-i \beta}$ of $R_{1} J$ and we can use the criterion (2-7) of Corollary 2.7 to determine for which values of $p$ the form $H_{\tau}$ has signature $(2,1)$. Alternatively, 

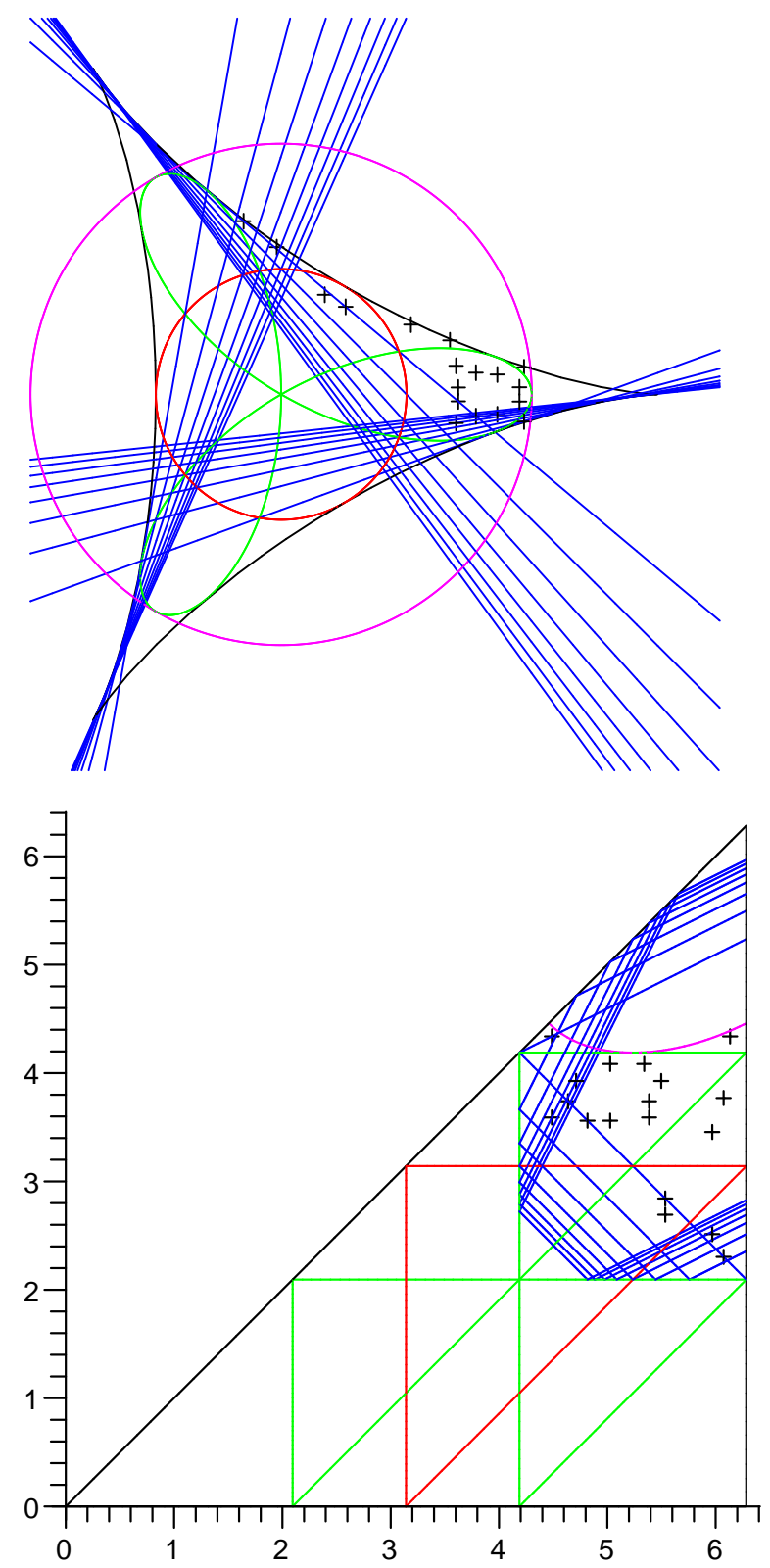

Figure 1. The points together with the trace parameter space (top) and the angle parameter space (bottom) for $p=3, \ldots, 10$.

we can use the angle pairs and check the linear conditions given in Section 2.6. We have plotted the points and parameter spaces for $p=3, \ldots, 10$ in the $\tau$-plane in Figure 1, top and the same thing in the angle parameter space in Figure 1, bottom. 
Proposition 3.3. Let $R_{1}$ have angle $\psi \in(0,2 \pi)$.

(i) Suppose that $\tau=\mathrm{m}(\phi)=-e^{-i \phi / 3}$. Then $H_{\tau}$ has signature $(2,1)$ if and only if $\sin (\psi+\phi / 2) \cos (\psi / 2-\phi / 2)>0$.

(ii) Suppose that $\tau=\mathrm{s}(\phi)=e^{2 i \phi / 3}+e^{-i \phi / 3}$. Then $H_{\tau}$ has signature $(2,1)$ if and only if $\sin (\psi / 2+\phi)>0$.

Proof. We insert the values of $\alpha$ and $\beta$ found above into Corollary 2.7 and simplify. In (i) we have $\alpha=\pi-\phi / 3$ and $\beta=\phi / 6$. This leads to $8 \sin (3 \pi / 2-\phi / 2+\psi / 2) \times$ $\sin (\phi / 4+\psi / 2) \sin (-3 \pi / 2+\phi / 4+\psi / 2)<0$. In (ii) we have $\alpha=\pi / 3-\phi / 3$ and $\beta=$ $2 \phi / 3$, leading to $8 \sin (\phi+\psi / 2) \sin (-\phi / 2+\pi / 2+\psi / 2) \sin (-\phi / 2-\pi / 2+\psi / 2)<$ 0 . Simplifying, this is equivalent to $\sin (\psi / 2+\phi)(\cos (\psi-\phi)+1)>0$. When $\cos (\psi-\phi)=-1$ and $|\psi| \leq 2 \pi / 3$ then $\sin (\psi / 2+\phi) \leq 0$. Therefore $H_{\tau}$ has signature $(2,1)$ if and only if $\sin (\psi / 2+\phi)>0$ as claimed.

We remark that the case $\cos (\psi-\phi)+1=0$ only occurs when $\tau=e^{2 i \psi / 3}-e^{-i \psi / 3}$. In this case $H_{\tau}$ has a repeated eigenvalue of 0 . These points correspond to the totally reducible groups $V_{1}$ and $V_{2}$ described in section 2.6.

For the sporadic groups we simply work by inspection. Here are the results:

\begin{tabular}{|l|l|l|l|l|l|l|}
\hline & $\alpha$ & $\beta$ & $-\alpha-\beta$ & $(2,1)$ & Degenerate & $(3,0)$ \\
\hline (iii) & $\pi / 3$ & $\pi / 12$ & $-5 \pi / 12$ & $p \geq 3$ & $p=2$ & \\
& $-\pi / 3$ & $5 \pi / 12$ & $-\pi / 12$ & $3 \leq p \leq 7$ & $p=2,8$ & $p \geq 9$ \\
(iv) & $\pi / 3$ & $\pi / 30$ & $-11 \pi / 30$ & $p \geq 3$ & $p=2$ & \\
& $-\pi / 3$ & $11 \pi / 30$ & $-\pi / 30$ & $3 \leq p \leq 19$ & $p=2,20$ & $p \geq 21$ \\
(v) & $\pi / 3$ & $7 \pi / 30$ & $-17 \pi / 30$ & $p \geq 3$ & $p=2$ & \\
& $-\pi / 3$ & $17 \pi / 30$ & $-7 \pi / 30$ & $3 \leq p \leq 6$ & $p=2$ & $p \geq 7$ \\
(vi) & $2 \pi / 7$ & $4 \pi / 7$ & $-6 \pi / 7$ & $4 \leq p \leq 6$ & $p=7$ & $p=2,3, p \geq 8$ \\
& $-2 \pi / 7$ & $-4 \pi / 7$ & $6 \pi / 7$ & $p \geq 3$ & & $p=2$ \\
(vii) & $2 \pi / 9$ & $13 \pi / 45$ & $-23 \pi / 45$ & $p \geq 2$ & & \\
(viii) & $-2 \pi / 9$ & $23 \pi / 45$ & $13 \pi / 45$ & $p=2,4$ & $p=3$ & $p \geq 5$ \\
& $2 \pi / 9$ & $31 \pi / 45$ & $-41 \pi / 45$ & $p \geq 3$ & & $p=2$ \\
(ix) & $-2 \pi / 9$ & $41 \pi / 45$ & $-31 \pi / 45$ & $4 \leq p \leq 29$ & $p=3,30$ & $p=2, p \geq 31$ \\
& $2 \pi / 9$ & $11 \pi / 63$ & $-25 \pi / 63$ & $p \geq 2$ & & \\
(x) & $-2 \pi / 9$ & $25 \pi / 63$ & $-11 \pi / 63$ & $p=2$ & $p=3$ & $p \geq 4$ \\
& $2 \pi / 9$ & $29 \pi / 63$ & $-43 \pi / 36$ & $4 \leq p \leq 41$ & $p=42$ & $p=2,3, p \geq 43$ \\
(xi) & $-2 \pi / 9$ & $43 \pi / 63$ & $-29 \pi / 63$ & $p \geq 4$ & $p=3$ & $p=2$ \\
& $2 \pi / 9$ & $47 \pi / 63$ & $-61 \pi / 63$ & $p \geq 3$ & & $p=2$ \\
& $-2 \pi / 9$ & $61 \pi / 63$ & $-47 \pi / 63$ & $4 \leq p \leq 8$ & $p=3$ & $p=2, p \geq 9$ \\
\hline
\end{tabular}




\section{Nondiscreteness results}

There are two simple ways to see that a subgroup of $\mathrm{PU}(2,1)$ is not discrete: finding an elliptic element of infinite order in the group, or finding a subgroup which stabilises a complex line, acting nondiscretely on it. Of course, these elementary facts can only be useful if one finds the appropriate element (word) or subgroup. We will present in this section some results of this nature.

4.1. Traces for certain words. We compute the traces of certain short words in our generators $R_{1}$ and $J$ (or $R_{1}, R_{2}, R_{3}$ ). These words seem relevant to us for experimental and/or historical reasons (see [Mostow 1980] and [Schwartz 2002] for instance). These traces can be computed in a straightforward manner from the generators, or by using the formulae from [Pratoussevitch 2005]. The result is shown here:

\begin{tabular}{|l|l|}
\hline$W$ & $\operatorname{tr}(W)$ \\
\hline$R_{1} J$ & $\tau$ \\
$J^{-1} R_{1}$ & $-e^{i \psi / 3} \bar{\tau}$ \\
$R_{1} R_{2} J^{-1}=\left(R_{1} J\right)^{2}$ & $\tau^{2}-2 \bar{\tau}$ \\
$J R_{2} R_{1}=\left(J^{-1} R_{1}\right)^{2}$ & $e^{2 i \psi / 3} \bar{\tau}^{2}+2 e^{-i \psi / 3} \tau$ \\
$R_{1} R_{2}$ & $e^{-2 i \psi / 3}+e^{i \psi / 3}\left(2-|\tau|^{2}\right)$ \\
$R_{1} R_{2} R_{3} R_{2}^{-1}$ & $e^{-2 i \psi / 3}+e^{i \psi / 3}\left(2-\left|\tau^{2}-\bar{\tau}\right|^{2}\right)$ \\
$R_{1} R_{2}^{-1} R_{3} R_{2}$ & $e^{-2 i \psi / 3}+e^{i \psi / 3}\left(2-\left|\tau^{2}+e^{i \psi} \bar{\tau}\right|^{2}\right)$ \\
$R_{1} R_{2}^{-1}$ & $1+2 \cos (\psi)+|\tau|^{2}$ \\
$R_{1} R_{2} R_{3}^{-1} R_{2}^{-1}$ & $1+2 \cos (\psi)+\left|\tau^{2}-\bar{\tau}\right|^{2}$ \\
$R_{1} R_{2}^{-1} R_{3}^{-1} R_{2}$ & $1+2 \cos (\psi)+\left|\tau^{2}+e^{i \psi} \bar{\tau}\right|^{2}$ \\
$R_{1} R_{2} R_{3}=\left(R_{1} J\right)^{3}$ & $3-3|\tau|^{2}+\tau^{3}$ \\
$R_{1} R_{2} R_{1} R_{2}^{-1} R_{1}^{-1} R_{2}^{-1}$ & $3+\left(|\tau|^{2}-1\right)\left(|\tau|^{4}+6|\tau|^{2}+2-2 \cos (\psi)\right)$ \\
\hline
\end{tabular}

We use this to prove nondiscreteness results, some valid for any value of $p$ like the following, and some depending on the value of $p$ (see last section for $p=3$ ).

Lemma 4.1. Let $R_{1}, R_{2}$ and $R_{3}$ be given by (2-6). If $\left|\tau^{2}-\bar{\tau}\right|>2$ then $R_{1} R_{2} R_{3} R_{2}^{-1}$ is loxodromic. If $\left|\tau^{2}-\bar{\tau}\right| \leq 2$ then $R_{1} R_{2} R_{3} R_{2}^{-1}$ is elliptic with eigenvalues $e^{-2 i \psi / 3}$, $-e^{i \psi / 3+2 i \theta^{\prime}}$ and $-e^{i \psi / 3-2 i \theta^{\prime}}$ where $\left|\tau^{2}-\bar{\tau}\right|=2 \cos \theta^{\prime}$.

Proof. A short computation shows that the trace of $R_{1} R_{2} R_{3} R_{2}^{-1}$ is

$$
\operatorname{tr}\left(R_{1} R_{2} R_{3} R_{2}^{-1}\right)=e^{i \psi / 3}\left(2-\left|\tau^{2}-\bar{\tau}\right|^{2}\right)+e^{-2 i \psi / 3} .
$$

Since all points of $L_{1}$ (the mirror of $R_{1}$ ) and of $R_{2}\left(L_{3}\right)$ (the mirror of $R_{2} R_{3} R_{2}^{-1}$ ) are $e^{-i \psi / 3}$ eigenvectors for $R_{1}$ and $R_{2} R_{3} R_{2}^{-1}$ respectively, we see that their intersection is an $e^{-2 i \psi / 3}$ eigenvector for $R_{1} R_{2} R_{3} R_{2}^{-1}$. If this point is outside $\mathbf{H}_{\mathbb{C}}^{2}$ 
then $R_{1} R_{2} R_{3} R_{2}^{-1}$ is loxodromic. On the other hand, if this point is inside complex hyperbolic space then $R_{1} R_{2} R_{3} R_{2}^{-1}$ is elliptic with eigenvalues $e^{-2 i \psi / 3},-e^{i \psi / 3+2 i \theta^{\prime}}$ and $-e^{i \psi / 3-2 i \theta^{\prime}}$ where $\left|\tau^{2}-\bar{\tau}\right|=2 \cos \theta^{\prime}$.

We now list $\left|\tau^{2}-\bar{\tau}\right|^{2}$ for the values of $\tau$ given in Proposition 3.1. It is clear that complex conjugating $\tau$ or multiplying by a cube root of unity does not affect this quantity. We also give the value of $\theta^{\prime}$ or indicate that $R_{1} R_{2} R_{3} R_{2}^{-1}$ is loxodromic.

\begin{tabular}{|l|l|ll|ll|}
\hline & $\tau$ & $|\tau|^{2}$ & $2 \theta$ & $\left|\tau^{2}-\bar{\tau}\right|^{2}$ & $2 \theta^{\prime}$ \\
\hline (i) & $-e^{-i \phi / 3}$ & 1 & $2 \pi / 3$ & $2+2 \cos (\phi)$ & $\phi$ \\
(ii) & $e^{2 i \phi / 3}+e^{-i \phi / 3}$ & $2+2 \cos (\phi)$ & $\phi$ & $2+2 \cos (\phi)$ & $\phi$ \\
(iii) & $e^{i \pi / 3}+e^{-i \pi / 6} 2 \cos (\pi / 4)$ & 3 & $\pi / 3$ & 2 & $\pi / 2$ \\
(iv) & $e^{i \pi / 3}+e^{-i \pi / 6} 2 \cos (\pi / 5)$ & $2+2 \cos (\pi / 5)$ & $\pi / 5$ & 3 & $\pi / 3$ \\
(v) & $e^{i \pi / 3}+e^{-i \pi / 6} 2 \cos (2 \pi / 5)$ & $2+2 \cos (3 \pi / 5)$ & $3 \pi / 5$ & 3 & $\pi / 3$ \\
(vi) & $e^{2 \pi i / 7}+e^{4 \pi i / 7}+e^{8 \pi i / 7}$ & 2 & $\pi / 2$ & 1 & $2 \pi / 3$ \\
(vii) & $e^{2 i \pi / 9}+e^{-i \pi / 9} 2 \cos (2 \pi / 5)$ & 2 & $\pi / 2$ & $2+2 \cos (2 \pi / 5)$ & $2 \pi / 5$ \\
(viii) & $e^{2 i \pi / 9}+e^{-i \pi / 9} 2 \cos (4 \pi / 5)$ & 2 & $\pi / 2$ & $2+2 \cos (4 \pi / 5)$ & $4 \pi / 5$ \\
(ix) & $e^{2 i \pi / 9}+e^{-i \pi / 9} 2 \cos (2 \pi / 7)$ & $2+2 \cos (\pi / 7)$ & $\pi / 7$ & $3+2 \cos (2 \pi / 7)$ & $\operatorname{lox}$. \\
(x) & $e^{2 i \pi / 9}+e^{-i \pi / 9} 2 \cos (4 \pi / 7)$ & $2+2 \cos (5 \pi / 7)$ & $5 \pi / 7$ & $3+2 \cos (4 \pi / 7)$ & irr. \\
(xi) & $e^{2 i \pi / 9}+e^{-i \pi / 9} 2 \cos (6 \pi / 7)$ & $2+2 \cos (3 \pi / 7)$ & $3 \pi / 7$ & $3+2 \cos (6 \pi / 7)$ & irr. \\
\hline
\end{tabular}

In case (ix) $\left|\tau^{2}-\bar{\tau}\right|^{2}>4$ and so $R_{1} R_{2} R_{3} R_{2}^{-1}$ is loxodromic.

In the last two cases $\left|\tau^{2}-\bar{\tau}\right|^{2}$ is less than 4 . Therefore, using Lemma 4.1 we see that $R_{1} R_{2} R_{3} R_{2}^{-1}$ is elliptic and we find the angle $\theta^{\prime}$ satisfying $2+2 \cos \left(2 \theta^{\prime}\right)=$ $\left|\tau^{2}-\bar{\tau}\right|^{2}=3+2 \cos (4 \pi / 7)$ and $3+2 \cos (6 \pi / 7)$, respectively. Using the theorem of Conway and Jones [Conway and Jones 1976, Theorem 7], we see that $\theta^{\prime}$ is not a rational multiple of $\pi$ and hence $R_{1} R_{2} R_{3} R_{2}^{-1}$ is elliptic of infinite order. Therefore, for these values of $\tau$, the group $\left\langle R_{1}, J\right\rangle$ is not discrete. This proves:

Corollary 4.2. Suppose that $\tau$ or $\bar{\tau}=e^{2 \pi i / 9}+e^{-\pi i / 9} 2 \cos (4 \pi / 7)$, or that $\tau$ or $\bar{\tau}=e^{2 \pi i / 9}+e^{-\pi i / 9} 2 \cos (6 \pi / 7)$. Then $R_{1} R_{2} R_{3} R_{2}^{-1}$ is elliptic of infinite order.

Hence when examining possible candidates for discrete groups it suffices to consider the values of $\tau$ given in parts (i) to (ix) of Proposition 3.1.

4.2. Triangle subgroups. We will systematically analyse triangle subgroups in order to find conditions for discreteness. By triangle subgroups we mean subgroups which fix a point in $\mathbb{C} P^{2}$; depending on the position of that point, the subgroup will stabilise a complex line (if the point is outside of $\overline{\mathbf{H}_{\mathbb{C}}^{2}}$ ), fix a point in $\mathbf{H}_{\mathbb{C}}^{2}$ or on its boundary. The two-generator group in question then acts as a hyperbolic, spherical, or Euclidean triangle group respectively, and the angles of the triangle action are obtained from the eigenvalues of the generators, see Proposition 4.4 below. 
We use the lists which answer the classical question of plane geometry: given three angles $\alpha, \beta$ and $\gamma$, when do the reflections in the sides of an $(\alpha, \beta, \gamma)$ triangle generate a discrete group?

In the Euclidean case it is easy to see that there are only four possibilities (see [Coxeter 1948]): $(\pi / 3, \pi / 3, \pi / 3),(\pi / 2, \pi / 4, \pi / 4)$ and $(\pi / 2, \pi / 3, \pi / 6)$, and $(2 \pi / 3, \pi / 6, \pi / 6)$.

In the case of a spherical triangle, the list is due to Schwarz, and we show it here arranged as in [Coxeter 1948]: colunar triangles appear together on one line, in increasing order of size.

\begin{tabular}{c}
$(\pi / 2, \pi / 2, p \pi / q)$ \\
\hline $\begin{array}{c}(\pi / 2, \pi / 3, \pi / 3),(\pi / 2,2 \pi / 3, \pi / 3),(\pi / 2,2 \pi / 3,2 \pi / 3) \\
(2 \pi / 3, \pi / 3, \pi / 3),(2 \pi / 3,2 \pi / 3,2 \pi / 3)\end{array}$ \\
\hline $\begin{array}{c}(\pi / 2, \pi / 3, \pi / 4),(\pi / 2,2 \pi / 3, \pi / 4),(\pi / 2, \pi / 3,3 \pi / 4),(\pi / 2,2 \pi / 3,3 \pi / 4) \\
(2 \pi / 3, \pi / 4, \pi / 4),(\pi / 3,3 \pi / 4, \pi / 4),(2 \pi / 3,3 \pi / 4,3 \pi / 4)\end{array}$ \\
$(\pi / 2, \pi / 3, \pi / 5),(\pi / 2,2 \pi / 3, \pi / 5),(\pi / 2, \pi / 3,4 \pi / 5),(\pi / 2,2 \pi / 3,4 \pi / 5)$ \\
$(2 \pi / 5, \pi / 3, \pi / 3),(3 \pi / 5,2 \pi / 3, \pi / 3),(2 \pi / 5,2 \pi / 3,2 \pi / 3)$ \\
$(2 \pi / 3, \pi / 5, \pi / 5),(\pi / 3,4 \pi / 5, \pi / 5),(2 \pi / 3,4 \pi / 5,4 \pi / 5)$ \\
$(\pi / 2,2 \pi / 5, \pi / 5),(\pi / 2,3 \pi / 5, \pi / 5),(\pi / 2,2 \pi / 5,4 \pi / 5),(\pi / 2,3 \pi / 5,4 \pi / 5)$ \\
$(3 \pi / 5, \pi / 3, \pi / 5),(2 \pi / 5,2 \pi / 3, \pi / 5),(2 \pi / 5, \pi / 3,4 \pi / 5),(3 \pi / 5,2 \pi / 3,4 \pi / 5)$ \\
$(2 \pi / 5,2 \pi / 5,2 \pi / 5),(3 \pi / 5,3 \pi / 5,2 \pi / 5)$ \\
$(2 \pi / 3, \pi / 3, \pi / 5),(\pi / 3, \pi / 3,4 \pi / 5),(2 \pi / 3,2 \pi / 3,4 \pi / 5)$ \\
$(4 \pi / 5, \pi / 5, \pi / 5),(4 \pi / 5,4 \pi / 5,4 \pi / 5)$ \\
$(\pi / 2,2 \pi / 5, \pi / 3),(\pi / 2,3 \pi / 5, \pi / 3),(\pi / 2,2 \pi / 5,2 \pi / 3),(\pi / 2,3 \pi / 5,2 \pi / 3)$ \\
$(3 \pi / 5,2 \pi / 5, \pi / 3),(2 \pi / 5,2 \pi / 5,2 \pi / 3),(3 \pi / 5,3 \pi / 5,2 \pi / 3)$
\end{tabular}

The list for hyperbolic triangles is due to Klimenko and Sakuma, following earlier work of Knapp [1968]:

Proposition 4.3 [Klimenko and Sakuma 1998, Lemma 2.1]. Suppose that the group generated by reflections in the sides of a hyperbolic triangle is discrete. Then the angles of the triangle appear on the following list:

(i) $\pi / p, \pi / q, \pi / r$ where $1 / p+1 / q+1 / r<1$;

(ii) $\pi / p, \pi / p, 2 \pi / r$ where $r$ is odd and $1 / p+1 / r<1 / 2$;

(iii) $\pi / p, \pi / 2,2 \pi / p$ where $p \geq 7$ is odd;

(iv) $\pi / p, \pi / 3,3 \pi / p$ where $p \geq 7$ is not divisible by 3 ;

(v) $\pi / p, \pi / p, 4 \pi / p$ where $p \geq 7$ is odd;

(vi) $2 \pi / p, 2 \pi / p, 2 \pi / p$ where $p \geq 7$ is odd;

(vii) $\pi / 7, \pi / 3,2 \pi / 7$. 
The case when two of the angles are $\pi / p$ and $\pi / q$ was proved by Knapp [1968, Theorem 2.3]. This list is the same as that above without case (vi). Knapp's theorem was rediscovered as Theorem 3.7 of [Mostow 1988] except that Mostow missed case (vii).

Given a group generated by reflections in the sides of a triangle (hyperbolic, Euclidean or spherical) we can consider the index 2 group of holomorphic motions. The product of a pair of reflections in sides that make an angle $\theta$ is an elliptic rotation through $2 \theta$. If we represent this map as a matrix in $\mathrm{SU}(2)$ or $\mathrm{SU}(1,1)$ the eigenvalues are $-e^{ \pm i \theta}$. This group of holomorphic motions is generated by $A$ and $B$ where $A, B, A B$ are elliptic rotations corresponding to products of pairs of reflections.

Proposition 4.4. For $j=1,2$ suppose that $B_{j}$ is a complex reflection in $\mathrm{SU}(2,1)$ with eigenvalues $e^{2 i \psi_{j} / 3}, e^{-i \psi_{j} / 3}, e^{-i \psi_{j} / 3}$ and mirror $L_{j}$. Then $L_{1} \cap L_{2}$ corresponds to an $e^{-i \psi_{1} / 3-i \psi_{2} / 3}$ eigenvector of $B_{1} B_{2}$. Suppose that $B_{1} B_{2}$ is elliptic and its other eigenvalues are $-e^{i \psi_{1} / 6+i \psi_{2} / 6 \pm i \psi_{3} / 2}$. Then on the orthogonal complement of $L_{1} \cap$ $L_{2}$ the group $\left\langle B_{1}, B_{2}\right\rangle$ acts as the holomorphic subgroup of $a\left(\psi_{1} / 2, \psi_{2} / 2, \psi_{3} / 2\right)$ triangle group.

Proof. We conjugate so that $L_{1} \cap L_{2}$ corresponds to the vector $(1,0,0)^{T}$. Then $B_{1}$ and $B_{2}$ are block diagonal matrices in $S(\mathrm{U}(1) \times \mathrm{U}(1,1))$ or $S(\mathrm{U}(1) \times \mathrm{U}(2))$. Scaling, we may suppose that they lie in $\mathrm{U}(1) \times \mathrm{SU}(1,1)$ or $\mathrm{U}(1) \times \mathrm{SU}(2)$. Then the action on the orthogonal complement of $L_{1} \cap L_{2}$ is given by the $2 \times 2$ unimodular matrices in the lower right hand block. These block matrices have eigenvalues $-e^{ \pm i \psi_{1} / 2},-e^{ \pm i \psi_{2} / 2},-e^{ \pm i \psi_{3} / 2}$ for $B_{1}, B_{2}, B_{1} B_{2}$ (up to maybe scaling by -1 ). The result follows from the remarks above.

For example, consider $R_{1}$ and $R_{2}$. Their eigenvalues are $e^{2 i \psi / 3}, e^{-i \psi / 3}, e^{-i \psi / 3}$. Scaling, we see that the eigenvalues of $-e^{-i \psi / 6} R_{1}$ and $-e^{-i \psi / 6} R_{2}$ are $-e^{i \psi / 2}$, $-e^{-i \psi / 2}$, and $-e^{-i \psi / 2}$. The eigenvalues of $R_{1} R_{2}$ are $-e^{i \psi / 3+2 i \theta},-e^{i \psi / 3-2 i \theta}$, and $e^{-2 i \psi / 3}$, so that the eigenvalues of $e^{-i \psi / 3} R_{1} R_{2}$ are $-e^{2 i \theta},-e^{-2 i \theta}$, and $e^{-i \psi}=$ $\left(-e^{-i \psi / 2}\right)\left(-e^{-i \psi / 2}\right)$. Therefore $\left\langle R_{1}, R_{2}\right\rangle$ acts on the orthognal complement of $L_{1} \cap L_{2}$ as the holomorphic subgroup of a $(\psi / 2, \psi / 2,2 \theta)$ triangle group.

Similarly, $R_{1}$ and $R_{2} R_{3} R_{2}^{-1}$ have a common eigenvector $L_{1} \cap R_{2}\left(L_{3}\right)$. When $R_{1} R_{2} R_{3} R_{2}^{-1}$ is elliptic the group $\left\langle R_{1}, R_{2} R_{3} R_{2}^{-1}\right\rangle$ acts on the orthogonal complement of $L_{1} \cap R_{2}\left(L_{3}\right)$ as a $\left(\psi / 2, \psi / 2,2 \theta^{\prime}\right)$ triangle group.

We can apply the result of Klimenko and Sakuma, Proposition 4.3 (in fact we only need the earlier version of [Knapp 1968]) to eliminate some of the cases, using the values for $2 \theta$ and $2 \theta^{\prime}$ given in Section 4.1. This gives the following:

Proposition 4.5. Suppose that $\psi=2 \pi / p$.

(i) If $p \geqslant 3$ and $\tau$ or $\bar{\tau}=e^{i \pi / 3}+e^{-i \pi / 6} 2 \cos (2 \pi / 5)$ then $\left\langle R_{1}, R_{2}\right\rangle$ is not discrete. 
(ii) If $p \neq 3,5$ and $\tau$ or $\bar{\tau}=e^{2 \pi i / 9}+e^{-\pi i / 9} 2 \cos (4 \pi / 5)$ then $\left\langle R_{1}, R_{2} R_{3} R_{2}^{-1}\right\rangle$ is not discrete.

\section{The two curves in parameter space}

In this section we determine exactly which points on the two curves from Proposition 3.1 correspond to discrete groups.

The first curve corresponds to the original groups from [Mostow 1980], where sufficient conditions for discreteness were found; these sufficient conditions were generalised in [Mostow 1986] (to a condition which Mostow calls $\Sigma$ INT, which also contains the groups from [Deligne and Mostow 1993]). A necessary condition, using triangle subgroups, was then found in [Mostow 1988], leaving only nine groups in dimension 2 and one in dimension 3 not covered by either of these criteria. In this same paper Mostow proved that all but three of these are discrete; the last three cases were shown to be discrete by Sauter [1990]. See the last two references for more details on the history of this question.

Determining which points of the second curve (or Sauter curve) correspond to discrete groups uses the result that every such group is a subgroup of a group on the Mostow curve, see Section 5.1 below. It then remains to discard the possibility that a discrete group on the Sauter curve could be a subgroup (of infinite index) of a nondiscrete group on the Mostow curve (Theorem 5.8). This is done by a careful analysis of triangle subgroups.

5.1. Sauter groups are subgroups of Mostow groups. In this section we prove that each group on the Sauter curve is conjugate to a subgroup of a group on the Mostow curve, but for a different value of $\psi$, the rotation angle of $R_{1}$. Recall that $\Gamma(\psi, \tau)$ denotes the group generated by $R_{1}$ and $J$, where $\psi$ is the rotation angle of $R_{1}$ and $\tau$ is the trace of $R_{1} J$.

Proposition 5.1. The group $\Gamma\left(\phi, e^{2 i \psi / 3}+e^{-i \psi / 3}\right)$ is conjugate to a subgroup of $\Gamma\left(\psi,-e^{-i \phi / 3}\right)$.

Proof. We begin by defining some elements of $\left\langle R_{1}, J\right\rangle$, with $\psi$ as rotation angle of $R_{1}$. These definitions follow Sauter.

$$
\begin{aligned}
& A_{1}=R_{2}^{-1} R_{1}^{-1} J=J^{-1}\left(J^{-1} R_{1}^{-1}\right)^{2} J=\left[\begin{array}{ccc}
-\tau & e^{i \psi / 3} \bar{\tau} & e^{-i \psi / 3} \\
e^{-i \psi / 3}\left(1-|\tau|^{2}\right) & \bar{\tau}^{2}-\tau & e^{-2 i \psi / 3} \bar{\tau} \\
0 & e^{2 i \psi / 3} & 0
\end{array}\right], \\
& A_{2}=J R_{2}^{-1} R_{1}^{-1}=\left(J^{-1} R_{1}^{-1}\right)^{2}, \quad A_{3}=J^{-1} R_{2}^{-1} R_{1}^{-1} J^{-1}=J\left(J^{-1} R_{1}^{-1}\right)^{2} J^{-1} .
\end{aligned}
$$

The result follows by noting that the subgroup $\left\langle A_{1}, J\right\rangle$ of $\left\langle R_{1}, J\right\rangle$ corresponds to the required parameters. Namely, if $\tau=-e^{-i \phi / 3}$ then $A_{1}$ is a complex reflection with angle $\phi$ (as can be seen by its trace), and $A_{1} J^{-1}$ has trace $e^{-i \psi / 3}\left(2-|\tau|^{2}\right)+$ 
$e^{2 i \psi / 3}=e^{-i \psi / 3}+e^{2 i \psi / 3}$. Note that we have replaced $J$ with $J^{-1}$, which is conjugate to $J$ (as in Sauter's isomorphisms).

In fact we can say more:

Lemma 5.2. $\left\langle A_{1}, A_{2}, A_{3}\right\rangle$ is a normal subgroup of $\left\langle R_{1}, J\right\rangle$.

Proof. If $|\tau|=1$ then $R_{i} R_{j} R_{i}=R_{j} R_{i} R_{j}$. Using this and $R_{2}=J R_{1} J^{-1}$, we find

$$
\begin{aligned}
& R_{1} A_{1} R_{1}^{-1}=R_{1} R_{2}^{-1} R_{1}^{-1} J R_{1}^{-1}=R_{2}^{-1} R_{1}^{-1} R_{2} R_{2}^{-1} J=A_{1}, \\
& R_{1} A_{2} R_{1}^{-1}=R_{1} J R_{2}^{-1} R_{1}^{-1} R_{1}^{-1}=R_{1} R_{2} J^{-1} J^{-1} R_{2}^{-1} R_{1}^{-1} J^{-1} J R_{2}^{-1} R_{1}^{-1}=A_{2}^{-1} A_{3} A_{2}, \\
& R_{1} A_{3} R_{1}^{-1}=R_{1} J^{-1} R_{2}^{-1} R_{1}^{-1} J^{-1} R_{1}^{-1}=J R_{2}^{-1} R_{1}^{-1}=A_{2} .
\end{aligned}
$$

(Compare the identities in Section 7 of [Parker 2006].) Moreover, from the definition of $A_{1}, A_{2}, A_{3}$ we have $J A_{i} J^{-1}=A_{i+1}$, taking the index $i$ mod 3. The lemma follows.

When $\tau=-e^{-i \phi / 3}$, Sauter considers the map $R_{1} \mapsto A_{2}, R_{2} \mapsto A_{1}, R_{3} \mapsto A_{3}$. That is, he considers the group for which the trace of the generator is $\bar{\tau}^{2}-2 \tau=$ $e^{2 i \phi / 3}+2 e^{-i \phi / 3}$ and the parameter is $e^{2 i \psi / 3}+e^{-i \psi / 3}$. This is a point on the curve of the second type. In fact, as Sauter is only interested in the case of Mostow groups, he only considers this map in the case when $\psi=2 \pi / 3$ [1990, Theorem 6.1]. In this case $e^{2 i \psi / 3}+e^{-i \psi / 3}=e^{i \pi / 9}$ which is an intersection point of the curves of Mostow and Sauter types (see Section 5.5).

The special case of groups on the Mostow curve with $\tau=1$ (or equivalently $\omega$ or $\bar{\omega})$ was considered in [Livné 1981]. Such groups have signature $(2,1)$ when $\psi<\pi / 2$. In [Parker 2006] we showed that Livné's groups contain subgroups that are triangle groups generated by involutions. This is another special case of Proposition 5.1.

Conversely, if $R_{1}, R_{2}$ and $R_{3}$ have the form (2-6) with $\tau=e^{2 i \phi / 3}+e^{-i \phi / 3}$ then the map

$$
Q=\left[\begin{array}{ccc}
e^{-i \phi / 3} & 0 & 0 \\
0 & 0 & e^{i \psi / 3+i \phi / 3} \\
0 & -e^{-i \psi / 3} & e^{2 i \phi / 3}+e^{-i \phi / 3}
\end{array}\right]
$$

satisfies

$$
Q R_{1} Q^{-1}=R_{1}, \quad Q R_{2} Q^{-1}=R_{3}, \quad Q R_{3} Q^{-1}=R_{3}^{-1} R_{2} R_{3} .
$$

Therefore $\left\langle R_{1}, R_{2}, R_{3}\right\rangle$ is a normal subgroup of $\left\langle Q, R_{1}, J\right\rangle$.

\subsection{The Mostow curves.}

Proposition 5.3. Suppose that $\psi=2 \pi / p$ and $\tau=-e^{-i \phi / 3}$. Then the following subgroups of $\left\langle R_{1}, J\right\rangle$ have a common fixed vector and on its orthogonal complement they act as (the holomorphic subgroup of) a triangle group as follows: 
(i) $\left\langle R_{1}, R_{2}\right\rangle$ acts as a $(\psi / 2, \psi / 2,2 \pi / 3)$ triangle group;

(ii) $\left\langle R_{1}, R_{2} R_{3} R_{2}^{-1}\right\rangle$ acts as a $(\psi / 2, \psi / 2, \phi)$ triangle group;

(iii) $\left\langle R_{1}, R_{2}^{-1} R_{3} R_{2}\right\rangle$ acts as a $(\psi / 2, \psi / 2, \pi-\psi-\phi)$ triangle group;

(iv) $\left\langle A_{1}, A_{2}\right\rangle=\left\langle R_{2}^{-1} R_{1}^{-1} J, J R_{2}^{-1} R_{1}^{-1}\right\rangle$ acts as a $(\phi / 2, \phi / 2, \psi)$ triangle group;

(v) $\left\langle A_{1}, A_{1} A_{3} A_{2}\right\rangle=\left\langle R_{2}^{-1} R_{1}^{-1} J,\left(R_{2}^{-1} R_{1}^{-1}\right)^{3}\right\rangle$ acts as a $(\phi / 2, \pi / 2-\psi / 2-\phi / 2$, $\pi / 2-3 \psi / 2)$ triangle group.

Proof. (i) The eigenvalues of $R_{1}$ and $R_{2}$ are $e^{2 i \psi / 3}, e^{-i \psi / 3}, e^{-i \psi / 3}$, and those of $R_{1} R_{2}$ are $-e^{i \psi / 3+2 i \pi / 3},-e^{i \psi / 3-2 i \pi / 3}, e^{-2 i \psi / 3}$. The result follows from Proposition 4.4 .

(ii) The eigenvalues of $R_{1}$ and $R_{2} R_{3} R_{2}^{-1}$ are $e^{2 i \psi / 3}, e^{-i \psi / 3}, e^{-i \psi / 3}$. The trace of $R_{1} R_{2} R_{3} R_{1}^{-1}$ is $e^{-2 i \psi / 3}-e^{i \psi / 3} 2 \cos (\phi)$. Therefore its eigenvalues are $-e^{i \psi / 3+i \phi}$, $-e^{i \psi / 3-i \phi}, e^{-2 i \psi / 3}$. The result follows from Proposition 4.4.

(iii) This is similar to (ii) except that

$\operatorname{tr}\left(R_{1} R_{2}^{-1} R_{3} R_{2}\right)=e^{-2 i \psi / 3}+e^{i \psi / 3} 2 \cos (\phi+\psi)=e^{-2 i \psi / 3}-e^{i \psi / 3} 2 \cos (\pi-\phi-\psi)$.

(iv) The eigenvalues of $A_{1}$ and $A_{2}$ are $e^{2 i \phi / 3}, e^{-i \phi / 3}, e^{-i \phi / 3}$. The trace of $A_{1} A_{2}$ is $e^{-2 i \phi / 3}-e^{i \phi / 3} 2 \cos (\psi)$. Therefore its eigenvalues are $e^{-2 i \phi / 3},-e^{i \psi+i \phi / 3}$ and $-e^{-i \psi+i \phi / 3}$. The result is again similar.

(v) Finally, the eigenvalues of $A_{1}$ are $e^{2 i \phi / 3}, e^{-i \phi / 3}, e^{-i \phi / 3}$ and those of $A_{1} A_{3} A_{2}=$ $\left(A_{1} J^{-1}\right)^{3}=\left(R_{2}^{-1} R_{1}^{-1}\right)^{3}$ are $e^{2 i \psi},-e^{-i \psi},-e^{-i \psi}$. The eigenvalues of $A_{3} A_{2}=$ $\left(A_{1}^{-1}\right)\left(A_{1} A_{2} A_{3}\right)$ are $e^{-2 i \phi / 3},-e^{i \psi+i \phi / 3},-e^{-i \psi+i \phi / 3}$. The result follows from Proposition 4.4.

Combining Proposition 5.3 with Proposition 4.3 we obtain:

Corollary 5.4. Suppose that $\psi=2 \pi / p$ and $\tau=-e^{-i \phi / 3}$. If $\left\langle R_{1}, J\right\rangle$ is discrete then either

(i) $\phi=2 \pi / q, \pi-\psi-\phi=\pi-2 \pi / p-2 \pi / q=2 \pi / r$ or

(ii) $\phi=2 \psi=4 \pi / q$ and $\pi-3 \psi=\pi-6 \pi / p=2 \pi / r$ where $p$ is odd.

Suppose that $p, q$ and $r$ are integers (or possibly $\infty$ ) in modulus at least 3, such that $1 / p+1 / q+1 / r=1 / 2$. Then up to permutation $p, q$ and $r$ take one of the 
values in this table:

\begin{tabular}{|r|rrrrrrrrrrrrrrr|}
\hline$p$ & 3 & 3 & 3 & 3 & 3 & 3 & 3 & 3 & 3 & 4 & 4 & 4 & 4 & 5 & 6 \\
$q$ & 3 & 4 & 5 & 6 & 7 & 8 & 9 & 10 & 12 & 4 & 5 & 6 & 8 & 5 & 6 \\
$r$ & -6 & -12 & -30 & $\infty$ & 42 & 24 & 18 & 15 & 12 & $\infty$ & 20 & 12 & 8 & 10 & 6 \\
\hline $2 p /(p-6)$ & -2 & -2 & -2 & -2 & -2 & -2 & -2 & -2 & -2 & -4 & -4 & -4 & -4 & -10 & $\infty$ \\
$2 q /(q-6)$ & -2 & -4 & -10 & $\infty$ & 14 & 8 & 6 & 5 & 4 & -4 & -10 & $\infty$ & 8 & -10 & $\infty$ \\
$2 r /(r-6)$ & 1 & $\frac{4}{3}$ & $\frac{5}{3}$ & 2 & $\frac{7}{3}$ & $\frac{8}{3}$ & 3 & $\frac{10}{3}$ & 4 & 2 & $\frac{20}{7}$ & 4 & 8 & 5 & $\infty$ \\
\hline
\end{tabular}

Theorem 5.5 [Mostow 1988; Sauter 1990]. Suppose $\psi=2 \pi / p$ and $\tau=-e^{-i \phi / 3}$, where

$$
\max \{-2 \psi, \psi-\pi\}<\phi<\min \{\pi+\psi, 2 \pi-2 \psi\}
$$

The group $\left\langle R_{1}, J\right\rangle$ corresponding to these parameters is discrete if and only if one of the following is true:

(i) $p=3,4,5,6,7,8,9,10,12,18$ or $\infty$ and $\phi=2 \pi / q, \pi-\psi-\phi=2 \pi / r$ for some integers $q, r$ (possibly infinity);

(ii) $p=5,7$ or 9 and $\phi=4 \pi / p$;

(iii) $p=5,7$ or 9 and $\phi=\pi-6 \pi / p$;

(iv) $p=15,24,42,-30$ or -12 and $\phi=2 \pi / 3$;

(v) $p=15,24,42,-30$ or -12 and $\phi=\pi / 3-2 \pi / p$.

Sauter [1990, Theorem 6.1] showed that the group from (v) with $\psi=2 \pi / p$ and $\phi=2 \pi / 3$ for $p=15,24,42,-30$ or -12 is conjugate to the group from (i) with $\psi=2 \pi / 3$ and $\phi=2 \pi / p$. This conjugation can be realised by the isomorphism identifying $A_{1}$ with $R_{1}$ and $J$ with $J^{-1}$. He also showed in [Sauter 1990, Theorem 6.2] shows that the group from (ii) with $\psi=2 \pi / p, \phi=4 \pi / p$ is conjugate to the group from (i) with $\psi=2 \pi / p$ and $\phi=\pi$. This isomorphism is slightly more complicated and involves sending a square root of $A_{1}$ to $R_{1}$ and $J$ to $J^{-1}$. The groups from (ii) and (iii) are conjugate as are the groups from (iv) and (v) via the map that fixes $R_{1}$ but sends $J$ to $J^{-1}$.

\subsection{The Sauter curves.}

Proposition 5.6. Suppose that $\psi=2 \pi / p$ and $\tau=e^{2 i \phi / 3}+e^{-i \phi / 3}$. Then the following subgroups of $\left\langle R_{1}, J\right\rangle$ have a common fixed vector and on its orthogonal complement they act as (the holomorphic subgroup of) a triangle group as follows:

(i) $\left\langle R_{1}, R_{2}\right\rangle$ acts as a $(\psi / 2, \psi / 2, \phi)$ triangle group;

(ii) $\left\langle R_{1}, R_{2} R_{3}\right\rangle$ acts as a $(\psi / 2, \pi / 2-\phi / 2-\psi / 2, \pi / 2-3 \phi / 2)$ triangle group. 
Proof. When $\tau=e^{2 i \phi / 3}+e^{-i \phi / 3}$ then $R_{1} R_{2} R_{3}=\left(R_{1} J\right)^{3}$ has one eigenvalue $e^{2 i \phi}$ and a repeated eigenvalue $-e^{-i \phi}$. Also, $R_{2} R_{3}$ has trace

$$
\operatorname{tr}\left(R_{2} R_{3}\right)=e^{i \psi / 3}\left(2-|\tau|^{2}\right)+e^{-2 i \psi / 3}=e^{i \psi / 3} 2 \cos (\phi)+e^{-2 i \psi / 3} .
$$

Hence $R_{2} R_{3}$ has eigenvalues $e^{-2 i \psi / 3},-e^{i \psi / 3+i \phi}$ and $e^{i \psi / 3-i \phi}$. Then $\mathbf{n}=L_{1} \cap L_{123}$ is a common eigenvector of $R_{1}$ and $R_{1} R_{2} R_{3}$, and hence of $R_{2} R_{3}$. The eigenvalues are $e^{-i \psi / 3},-e^{-i \phi}$ and $-e^{i \psi / 3-i \phi}$ respectively. Thus on the orthogonal complement of $\mathbf{n}$ the group $\left\langle R_{1}, R_{2} R_{3}\right\rangle$ acts as a $(\psi / 2, \pi / 2-\phi / 2-\psi / 2, \pi / 2-3 \phi / 2)$ triangle group.

We again use Proposition 4.3 to eliminate all but finitely many points.

Corollary 5.7. Suppose that $\psi=2 \pi / p$ and $\tau=e^{2 i \phi / 3}+e^{-i \phi / 3}$. If $\left\langle R_{1}, J\right\rangle$ is discrete then $\phi=2 \pi / q$ where either

(i) $\pi-\psi-\phi=\pi-2 \pi / p-2 \pi / q=2 \pi / r$ and $\pi-3 \phi=\pi-6 \pi / q=2 \pi / s$ or

(ii) $p=3$ and $\pi / 3-\phi=\pi / 3-2 \pi / q=2 \pi / s$.

Proof. Using $\psi=2 \pi / p$ in $\left\langle R_{1}, R_{2}\right\rangle$ and Proposition 4.3, we see that either $\phi=$ $2 \pi / q$ or $4 \pi / p$ and in the latter case $p$ is odd.

Assume that $\phi=2 \pi / q$. Then $\left\langle R_{1}, R_{2} R_{3}\right\rangle$ acts as a $(\pi / p, \pi / 2-\pi / p-\pi / q$, $\pi / 2-3 \pi / q)$ triangle group. From Proposition 4.3 we see that either $\pi / 2-\pi / p-$ $\pi / q$ or $\pi / 2-3 \pi / q$ has the form $\pi / r$. The result follows from the table above. Note that in (ii) we have $3 \pi / s=\pi / 2-3 \pi / q=3(\pi / 2-\pi / 3-\pi / q)$ and, when $s$ is not divisible by 3 we are in case (iv) of Proposition 4.3.

If $\phi=4 \pi / p$ then $\left\langle R_{1}, R_{2} R_{3}\right\rangle$ acts as a $(\pi / p, \pi / 2-3 \pi / p, \pi / 2-6 \pi / p)$ triangle group. By inspection we see that the only possible values of $p$ satisfying Proposition 4.3 are $p=6,8,9,10,12,14,18$. Of these, only $p=9$ is odd. We now eliminate this case.

Suppose that $\psi=2 \pi / 9$ and $\phi=4 \pi / 9$. That is:

$$
R_{1}=\left[\begin{array}{ccc}
e^{4 \pi i / 27} & e^{2 \pi i / 27} 2 \cos (2 \pi / 9) & -2 \cos (2 \pi / 9) \\
0 & e^{-2 \pi i / 27} & 0 \\
0 & 0 & e^{-2 \pi i / 27}
\end{array}\right]
$$

We calculate

$$
\left(R_{1} R_{2}\right)^{6}=\left[\begin{array}{ccc}
e^{4 \pi i / 9}(2 \cos (2 \pi / 9)-1) & -e^{16 \pi i / 27}(2 \cos (4 \pi / 9)+1) & 0 \\
e^{8 \pi i / 27}(2 \cos (4 \pi / 9)+1) & -e^{4 \pi i / 9} 2 \cos (2 \pi / 9) & 0 \\
0 & 0 & e^{-8 \pi i / 9}
\end{array}\right] .
$$


The eigenvalues of $\left(R_{1} R_{2}\right)^{6}$ are $e^{16 \pi i / 9}, e^{-8 \pi i / 9}, e^{-8 \pi i / 9}$. Thus $\left(R_{1} R_{2}\right)^{6}$ is a complex reflection with angle $8 \pi / 3$, that is $2 \pi / 3$. Also

$$
\operatorname{tr}\left(\left(R_{1} R_{2}\right)^{6} J\right)=-e^{16 \pi i / 27}(2 \cos (4 \pi / 9)+1) .
$$

This trace does not appear on our list of possible values of $\tau$. Therefore $\left\langle R_{1}, J\right\rangle$ is not discrete. Indeed, we may calculate that

$$
\operatorname{tr}\left(\left(R_{1} R_{2}\right)^{6}\left(R_{2} R_{3}\right)^{6}\right)=e^{8 \pi i / 9}(2 \cos (2 \pi / 9)-1-2 \cos (4 \pi / 9))+e^{-16 \pi i / 9} .
$$

Now $2 \cos (2 \pi / 9)-1-2 \cos (4 \pi / 9)$ lies in $(-2,2)$, but is not equal to twice the cosine of a rational multiple of $\pi$ (for example this follows from the theorem of Conway and Jones). Hence $\left(R_{1} R_{2}\right)^{6}\left(R_{2} R_{3}\right)^{6}$ is elliptic of infinite order.

We now consider the points we have not eliminated by Corollary 5.7. By inspection, we see that each of these groups is a subgroup of one of the discrete groups from Theorem 5.5. Therefore we have proven:

Theorem 5.8. Suppose that $\psi=2 \pi / p$ and $\tau=e^{2 i \phi / 3}+e^{-i \phi / 3}$ where $-\pi / p<$ $\phi<\pi-\pi / p$. The group $\left\langle R_{1}, J\right\rangle$ is discrete if and only if $\phi=2 \pi / q$ where either

(i) $q=3,4,5,6,7,8,9,10,12,18$ or $\infty$ and $\pi-2 \pi / p-2 \pi / q=2 \pi / r$ for some integer $r$;

(ii) $p=3$ and $q=15,24,42,-30$ or -12 .

Note that (i) includes the case where $p=2$ and $r=-q$ (where $q \geqslant 5$ ), which is Proposition 4.5 of [Parker 2008], and the case where $p=3$ and $q=$ $3,4,5,6,7,8,9,10,12,18$ or $\infty$.

5.4. An example of a discrete group on the Sauter curve. Paupert [Paupert 2005, $\S 5.4 .1]$ considered the group with $p=3$ and angle parameters $(5 \pi / 3,4 \pi / 3)$. Writing $R_{1} J$ as a unimodular matrix, these angle parameters translate to eigenvalues $1, e^{i \pi / 3}$ and $e^{-i \pi / 3}$. (The fixed point of $R_{1} J$ corresponds to the $e^{i \pi / 3}$-eigenspace.) Hence the trace of $R_{1} J$ is $\tau=1+e^{i \pi / 3}+e^{-i \pi / 3}=2$. This appears on the list of discrete groups. In this case,

$$
\begin{gathered}
R_{1}=\left[\begin{array}{ccc}
e^{4 \pi i / 9} & 2 & -2 e^{2 \pi i / 9} \\
0 & e^{-2 \pi i / 9} & 0 \\
0 & 0 & e^{-2 \pi i / 9}
\end{array}\right], \quad R_{2}=\left[\begin{array}{ccc}
e^{-2 \pi i / 9} & 0 & 0 \\
-2 e^{2 \pi i / 9} & e^{4 \pi i / 9} & 2 \\
0 & 0 & e^{-2 \pi i / 9}
\end{array}\right], \\
R_{3}=\left[\begin{array}{ccc}
e^{-2 \pi i / 9} & 0 & 0 \\
0 & e^{-2 \pi i / 9} & 0 \\
2 & -2 e^{2 \pi i / 9} & e^{4 \pi i / 9}
\end{array}\right] .
\end{gathered}
$$


We can easily check that an $e^{i \pi / 3}$-eigenvector of $R_{1} J$ is

$$
\left[\begin{array}{c}
e^{5 \pi i / 9} \\
1 \\
e^{-5 \pi i / 9}
\end{array}\right]
$$

which is negative.

We now give another, more direct, way to see that this group is discrete. Using Proposition 2.8 we see that $\left\langle R_{1}, R_{2}, R_{3}\right\rangle$ may be conjugated in $\mathrm{PU}(2,1)$ so that their matrix entries lie in $\mathbb{Z}\left[e^{2 \pi i / 3}\right]$, the Eisenstein integers. Since this is a discrete subring of $\mathbb{C}$ the group is automatically discrete.

5.5. The two curves are related for $\boldsymbol{p}=3$. In Section 5.1 we have seen that each group on the Sauter curve is a subgroup of a group on the Mostow curve, but for a different value of $\psi$ (the angle of rotation of the generators). In the special case where $\psi=2 \pi / 3$, we can apply this twice to see that each group on the Sauter curve is a subgroup of a group on the Mostow curve, for the same value of $\psi$. Moreover, Theorem 5.8 tells us that the Sauter subgroup in question is discrete only if the larger Mostow group is discrete. In other words, in terms of discrete groups, the two curves are the same; this is visible on Figure 3. The precise statement is the following:

Proposition 5.9. The group $\Gamma\left(2 \pi / 3, e^{2 i \phi / 3}+e^{-i \phi / 3}\right)$ is conjugate to a subgroup of $\Gamma\left(2 \pi / 3,-e^{-i \phi / 3}\right)$.

Proof. We have seen in Section 5.1 that $\Gamma\left(\phi, e^{2 i \psi / 3}+e^{-i \psi / 3}\right)$ is conjugate to a subgroup of $\Gamma\left(\psi,-e^{-i \phi / 3}\right)$. Applying this with $\psi=2 \pi / 3$, we obtain that $\Gamma\left(\phi, e^{4 i \pi / 9}+e^{-2 i \pi / 9}\right)$ is conjugate to a subgroup of $\Gamma\left(2 \pi / 3,-e^{-i \phi / 3}\right)$. Now the point is that $e^{4 i \pi / 9}+e^{-2 i \pi / 9}=e^{i \pi / 9}=-e^{-8 i \pi / 9}$, so that the former group (of Sauter type) is also of Mostow type, and we can therefore repeat the process. This tells us that $\Gamma\left(8 \pi / 3, e^{2 i \phi / 3}+e^{-i \phi / 3}\right)$ is conjugate to a subgroup of $\Gamma\left(2 \pi / 3,-e^{-i \phi / 3}\right)$. Note that $8 \pi / 3=2 \pi / 3 \bmod 2 \pi$.

In fact we can say more and identify the corresponding subgroups in terms of generators: $\left\langle\left(R_{1} R_{2}\right)^{2}, J\right\rangle$ is a subgroup of $\left\langle A_{1}, J\right\rangle$, which is in turn a subgroup of $\left\langle R_{1}, J\right\rangle$ (see Section 5.1), and these various subgroups correspond to the values of the parameters described above.

Indeed, when $p=3$ and $|\tau|=1$, we have $\operatorname{tr}\left(\left(R_{1} R_{2}\right)^{2}\right)=e^{16 \pi i / 9}+2 e^{-8 \pi i / 9}$. Therefore $\left(R_{1} R_{2}\right)^{2}$ is a complex reflection with angle $e^{24 \pi i / 9}=e^{2 \pi i / 3}$. We also have $\operatorname{tr}\left(\left(R_{1} R_{2}\right)^{2} J\right)=\bar{\tau}^{2}+2 \cos (2 \pi / 3) \tau$. When $\tau=-e^{-i \phi / 3}$ then this trace is $e^{2 i \phi / 3}+e^{-i \phi / 3}$ as required. 


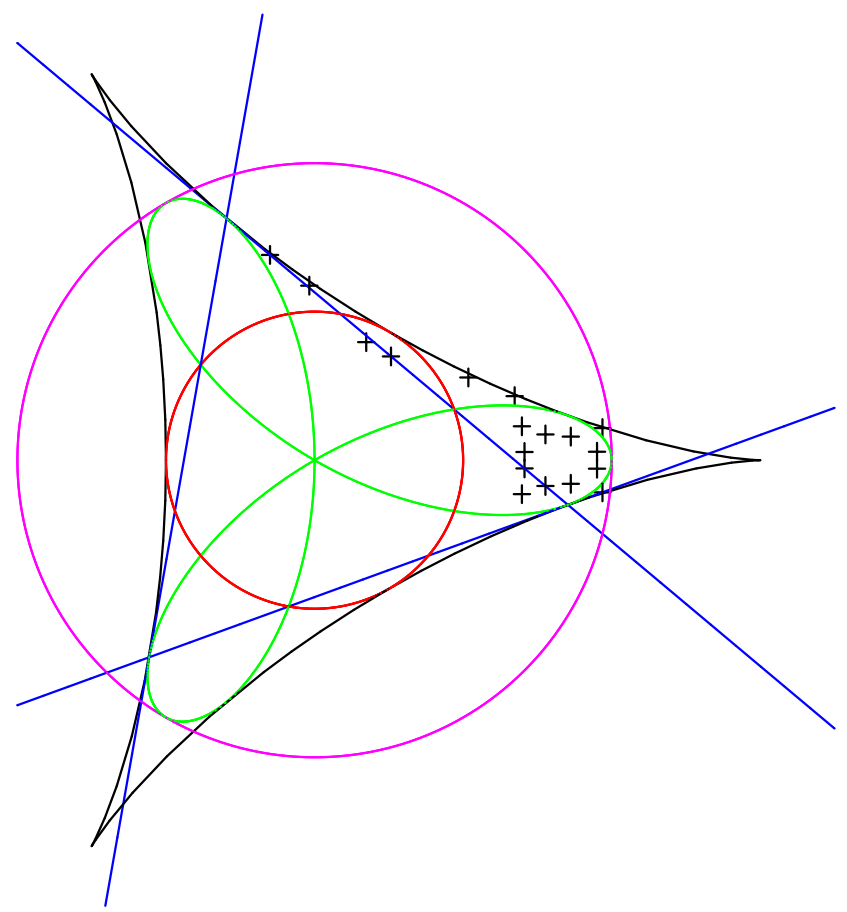

Figure 2. The sporadic points together with the trace parameter space for $p=3$.

\section{Sporadic groups generated by complex reflections of order 3}

We summarise the results in this section in the following theorem. Recall that the sporadic values of $\tau$ are denoted $\sigma(\phi, \eta)=e^{2 i \phi}+e^{-i \phi} 2 \cos (\eta)$, except for $\sigma( \pm 2 \pi / 7)=e^{ \pm 2 \pi i / 7}+e^{ \pm 4 \pi i / 7}+e^{ \pm 8 \pi i / 7}$.

Theorem 6.1. Suppose that $\psi=2 \pi / 3$. Then the groups associated to the sporadic values of $\tau$ have the following properties:

(i) $\Gamma(2 \pi / 3, \tau)$ lies in $\mathrm{SU}(2,1)$ unless $\tau=\sigma(\pi / 9,4 \pi / 7)$ or $\sigma(2 \pi / 7)$.

(ii) $\Gamma(2 \pi / 3, \tau)$ fixes a point of $\mathbf{H}_{\mathbb{C}}^{2}$ if and only if $\tau=\sigma(-\pi / 9, \eta)$ where $\eta=$ $2 \pi / 5,4 \pi / 5,4 \pi / 7,6 \pi / 7$.

(iii) $\Gamma(2 \pi / 3, \tau)$ preserves a complex line if and only if $\tau=\sigma(-\pi / 9,2 \pi / 7)$.

(iv) $\Gamma(2 \pi / 3, \tau)$ is contained in an arithmetic lattice in $\mathrm{SU}(2,1)$ if and only if $\tau=\sigma(-2 \pi / 7)$.

(v) If $\tau \in\{\sigma( \pm \pi / 6,2 \pi / 5), \sigma(\pi / 9,4 \pi / 5), \sigma(-\pi / 9,4 \pi / 7), \sigma( \pm \pi / 9,6 \pi / 7)\}$ then $\Gamma(2 \pi / 3, \tau)$ is not discrete. 


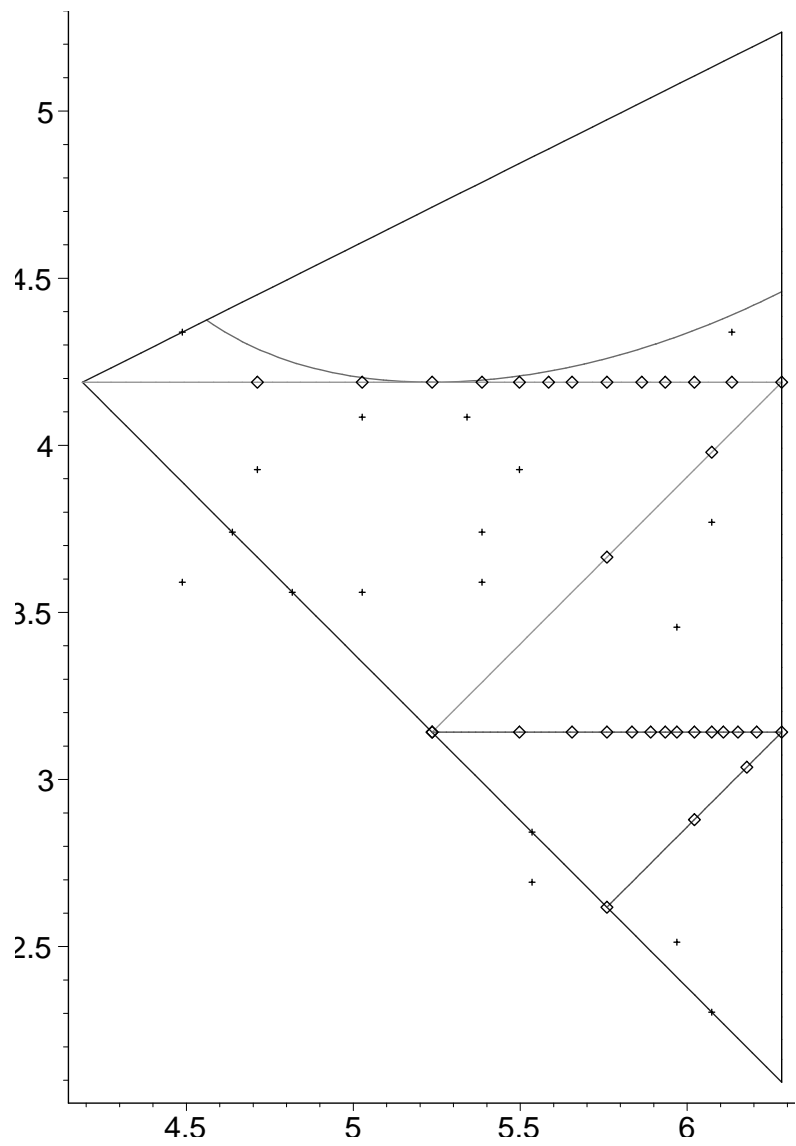

Figure 3. The sporadic points (crosses) together with the angle parameter space for $p=3$. We have also included all discrete points (diamonds) on the two curves.

6.1. Which sporadic points are in the parameter space? This was determined earlier for all values of $p$ (see Table 2). For $p=3$, the situation is the following:

- $H_{\tau}$ has signature $(2,1)$ for the following sporadic values of $\tau$ :

$$
\begin{array}{cccc}
\sigma( \pm \pi / 6, \pi / 4), & \sigma( \pm \pi / 6, \pi / 5), & \sigma( \pm \pi / 6,2 \pi / 5), & \sigma(-2 \pi / 7) \\
\sigma(\pi / 9,2 \pi / 5), & \sigma(\pi / 9,4 \pi / 5), & \sigma(\pi / 9,2 \pi / 7), & \sigma(\pi / 9,6 \pi / 7) .
\end{array}
$$

- $H_{\tau}$ is degenerate (signature $(2,0)$ or $(1,1)$, see below) for $\tau=\sigma(-\pi / 9, \eta)$ where $\eta=2 \pi / 5,4 \pi / 5,2 \pi / 7,4 \pi / 7$ or $6 \pi / 7$. The corresponding five points are on the boundary of our parameter space.

- $H_{\tau}$ is positive definite when $\tau=\sigma(\pi / 9,4 \pi / 7)$ and $\tau=\sigma(2 \pi / 7)$. The corresponding two points are outside of our parameter space. 

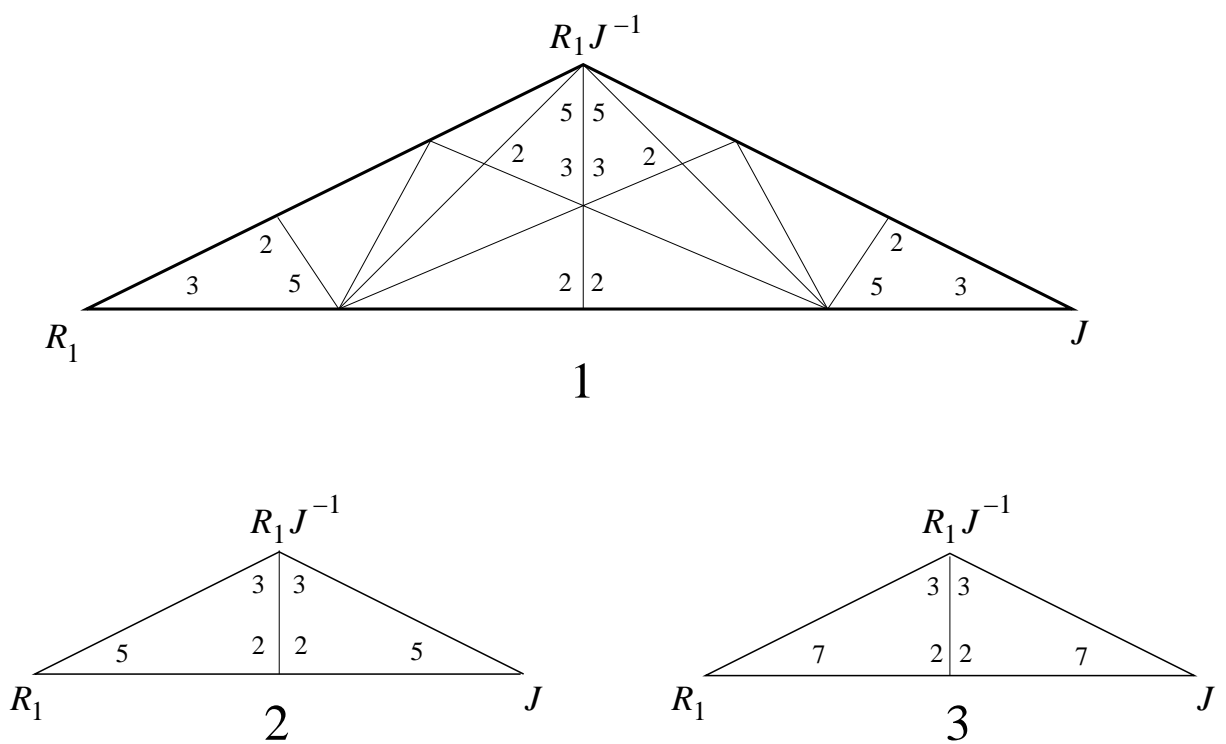

Figure 4. Schematic picture of triangle group action when $\tau=$ $\sigma(-\pi / 9, \eta)$ for $\eta=2 \pi / 5(2), 4 \pi / 5(1), 2 \pi / 7$ (3).

6.2. Reducible sporadic groups. These groups correspond to $\tau=\sigma(-\pi / 9, \eta)$ where $\eta=2 \pi / 5,4 \pi / 5,2 \pi / 7,4 \pi / 7$ or $6 \pi / 7$ and have a common eigenvector

$$
\left[\begin{array}{l}
1 \\
1 \\
1
\end{array}\right] \text {. }
$$

As in Proposition 4.4 we analyse their action on the orthogonal complement of this vector. In this case, normalising $R_{1}$ for convenience as $-e^{-i \pi / 9} R_{1}$, the eigenvalues on this complement are $-e^{i \pi / 3}$ and $-e^{-i \pi / 3}$ for $R_{1},-e^{i \pi / 3}$ and $-e^{-i \pi / 3}$ for $J$, and $-e^{i \eta}$ and $-e^{-i \eta}$ for $R_{1} J$. Therefore the group $\left\langle R_{1}, J\right\rangle$ acts as (the holomorphic subgroup of) a $(\pi / 3, \pi / 3, \eta)$ triangle group. For $\eta=2 \pi / 5$ or $4 \pi / 5$, this is in fact a (finite) $(2,3,5)$ triangle group; for $\eta=2 \pi / 7$, it is a discrete hyperbolic $(2,3,7)$ triangle group. For $\eta=4 \pi / 7$ or $6 \pi / 7$, it is a nondiscrete spherical triangle group; in fact we already know that these two groups are nondiscrete from Corollary 4.2, as $R_{1} R_{2} R_{3} R_{2}^{-1}$ is elliptic of infinite order.

In Figure 4 we draw a schematic picture of the triangle group action induced by the generators in the three discrete cases.

6.3. Nondiscrete irreducible sporadic groups. The trace of $R_{1} R_{2}^{-1}$ is

$$
\operatorname{tr}\left(R_{1} R_{2}^{-1}\right)=2 \cos (\psi)+|\tau|^{2}+1 .
$$


When $\psi=2 \pi / 3$ we have

$$
\operatorname{tr}\left(R_{1} R_{2}^{-1}\right)=|\tau|^{2}=2+2 \cos (2 \theta)
$$

where $2 \theta$ is given in the table just before Proposition 3.1. Evaluating in each case we can find whether $R_{1} R_{2}^{-1}$ is loxodromic, parabolic or elliptic and, in the latter case, whether or not it has finite order. This enables us to eliminate one pair of complex conjugate $\tau$ (see also Proposition 4.5):

Lemma 6.2. Suppose that $\psi=2 \pi / 3$ and $\tau=\sigma( \pm \pi / 6,2 \pi / 5)$. Then $R_{1} R_{2}^{-1}$ is elliptic of infinite order.

Proof. We know that

$$
\operatorname{tr}\left(R_{1} R_{2}^{-1}\right)=|\tau|^{2}=1+4 \cos ^{2}(2 \pi / 5)=3+2 \cos (4 \pi / 5) .
$$

Since $R_{1} R_{2}^{-1}$ has real trace it must have eigenvalues $e^{i \Theta}, e^{-i \Theta}$ and 1 . Hence its trace is $1+2 \cos (\Theta)$. In other words,

$$
2 \cos (\Theta)=2-2 \cos (\pi / 5) .
$$

Using the theorem of Conway and Jones [1976, Theorem 7], we see that $\Theta$ is not a rational multiple of $\pi$. This proves the result.

The proof of the following lemma is similar to the proof of Lemma 4.1, once we know that

$$
\operatorname{tr}\left(R_{1} R_{2}^{-1} R_{3} R_{2}\right)=e^{i \psi / 3}\left(2-\left|\tau^{2}-e^{i \psi} \bar{\tau}\right|^{2}\right)+e^{-2 i \psi / 3} .
$$

Lemma 6.3. Let $R_{1}, R_{2}$ and $R_{3}$ be given by (2-6). If $\left|\tau^{2}+\bar{\tau} e^{i \psi}\right|>2$ then $R_{1} R_{2}^{-1} R_{3} R_{2}$ is loxodromic. If $\left|\tau^{2}-\bar{\tau}\right| \leq 2$ then $R_{1} R_{2}^{-1} R_{3} R_{2}$ is elliptic with eigenvalues $e^{-2 i \psi / 3},-e^{i \psi / 3+2 i \Theta}$ and $-e^{i \psi / 3-2 i \Theta}$ where $\left|\tau^{2}+\bar{\tau} e^{i \psi}\right|=2 \cos \Theta$.

Corollary 6.4. Suppose that $\psi=2 \pi / 3$ and $\tau=\sigma(\pi / 9,4 \pi / 5)$. Then $R_{1} R_{2}^{-1} R_{3} R_{2}$ is elliptic of infinite order.

Proof. When $\psi=2 \pi / 3$ and $\tau=\sigma(\pi / 9,4 \pi / 5)$ we see that

$$
\left|\tau^{2}+\bar{\tau} e^{i \psi}\right|^{2}=6-\sqrt{5}
$$

Hence $\left|\tau^{2}+\bar{\tau} e^{i \psi}\right|=2 \cos \Theta$ where $\Theta$ is not a rational multiple of $\pi$.

6.4. Arithmeticity of sporadic groups. We begin by applying Proposition 2.8 to show that when $\tau=\sigma(-2 \pi / 7)$ the group $\left\langle R_{1}, R_{2}, R_{3}\right\rangle$ is contained in an arithmetic lattice in $\mathrm{SU}(2,1)$. In particular, putting $\psi=2 \pi / 3$ into the expression in Equation (2-8) for $2 \sin (\pi / 3) C H_{\tau} C^{-1}$, where $C=\operatorname{diag}\left(e^{-2 \pi i / 9}, 1, e^{2 \pi i / 9}\right)$, we obtain:

$$
\sqrt{3} C H_{\tau} C^{-1}=\left[\begin{array}{ccc}
3 & -(3+i \sqrt{3}) \tau / 2 & (3+i \sqrt{3}) \bar{\tau} / 2 \\
-(3-i \sqrt{3}) \bar{\tau} / 2 & 3 & -(3+i \sqrt{3}) \tau / 2 \\
(3-i \sqrt{3}) \tau / 2 & -(3-i \sqrt{3}) \bar{\tau} / 2 & 3
\end{array}\right] .
$$


Proposition 6.5. Let $R_{1}, R_{2}$ and $R_{3}$ be complex reflections with angle $\psi=2 \pi / 3$ so that the group $\left\langle R_{1}, R_{2}, R_{3}\right\rangle$ has parameter $\tau=\sigma(-2 \pi / 7)=(-1-i \sqrt{7}) / 2$. Then $\left\langle R_{1}, R_{2}, R_{3}\right\rangle$ is contained in an arithmetic lattice in $\mathrm{SU}(2,1)$ and hence is discrete.

Proof. We have $e^{i \psi}=(-1+i \sqrt{3}) / 2$ and $\tau=(-1-i \sqrt{7}) / 2$. Both of these are algebraic integers in the field $\mathbb{Q}(\sqrt{21}, i \sqrt{3})$. In particular, using Proposition 2.8, we can ensure that the matrix entries of all elements of $\left\langle R_{1}, R_{2}, R_{3}\right\rangle$ are algebraic integers in $\mathbb{Q}(\sqrt{21}, i \sqrt{3})$. This field is a totally imaginary quadratic extension of the totally real number field $\mathbb{Q}(\sqrt{21})$. The only nontrivial Galois conjugation in $\mathbb{Q}(\sqrt{21})$ sends $\sqrt{21}$ to $-\sqrt{21}$. This is compatible with $g$, the Galois conjugation in $\mathbb{Q}(\sqrt{21}, i \sqrt{3})$ fixing $(-1+i \sqrt{3}) / 2$ and sending $\tau=(-1-i \sqrt{7}) / 2$ to $\bar{\tau}=(-1+i \sqrt{7}) / 2$. The matrix $\sqrt{3} C H_{\tau} C^{-1}$ has entries in the ring of integers of $\mathbb{Q}(\sqrt{21}, i \sqrt{3})$ and signature $(2,1)$. The Galois conjugation $g$ sends $\sqrt{3} H_{\tau}$ to $\sqrt{3} C H_{\bar{\tau}} C^{-1}$. But we know that the point $\bar{\tau}$ lies outside our parameter space and so corresponds to a group for which the signature is $(3,0)$. Therefore, using standard arguments (for example [McReynolds 2006] or Proposition 4.3 of [Parker 2008]), we see that this group is contained in an arithmetic lattice in $\mathrm{SU}(2,1)$ and hence discrete.

There are ten more sporadic groups with signature $(2,1)$. The goal of the rest of this section is to show that none of them are contained in an arithmetic lattice in $\mathrm{SU}(2,1)$.

Proposition 6.6. Let $R_{1}, R_{2}$ and $R_{3}$ be complex reflections with angle $\psi=2 \pi / 3$ so that the group $\left\langle R_{1}, R_{2}, R_{3}\right\rangle$ has parameter $\tau \neq \sigma(-2 \pi / 7)=(-1-i \sqrt{7}) / 2$. Then $\left\langle R_{1}, R_{2}, R_{3}\right\rangle$ is not contained in an arithmetic lattice in $\mathrm{SU}(2,1)$.

The method of proof will be very similar to the proof of Proposition 6.6 and we give an outline. In each case we conjugate (multiples of) $H_{\tau}$ and $R_{j}$ so that their matrix entries are algebraic integers in a number field $E$. In each case the number field $E$ will be a purely imaginary quadratic extension of a totally real field $F$. The following lemma lists the values of $\tau$ and the number fields. It is easy to verify and we leave the details to the reader. Note that in parts (i) and (ii) we conjugate by $C$ and in parts (iii) and (iv) we conjugate by $C^{-1}$.

Lemma 6.7. Suppose that $R_{1}, R_{2}$ and $R_{3}$ are given by (2-6) with $\psi=2 \pi / 3$ and that $C=\operatorname{diag}\left(e^{-2 \pi i / 9}, 1, e^{2 \pi i / 9}\right)$.

(i) If $\tau=\sigma(\pi / 6, \pi / 4)$ or $\sigma(-\pi / 6, \pi / 4)$ then the entries of $\sqrt{3} C H_{\tau} C^{-1}$ and $e^{2 \pi i / 9} C R_{j} C^{-1}$, for $j=1,2,3$, are algebraic integers in the number field $\mathbb{Q}(\sqrt{6}, i \sqrt{3})$. 
(ii) If $\tau=\sigma(\pi / 6, \pi / 5), \sigma(\pi / 6,2 \pi / 5), \sigma(-\pi / 6, \pi / 5)$ or $\sigma(-\pi / 6,2 \pi / 5)$ then the entries of $\sqrt{3} C H_{\tau} C^{-1}$ and $e^{2 \pi i / 9} C R_{j} C^{-1}$, for $j=1,2,3$, are algebraic integers in the number field $\mathbb{Q}(\sqrt{3}, \sqrt{5}, i)$.

(iii) If $\tau=\sigma(\pi / 9,2 \pi / 5)$ or $\sigma(\pi / 9,4 \pi / 5)$ then the entries of $\sqrt{3} C^{-1} H_{\tau} C$ and $e^{2 \pi i / 9} C^{-1} R_{j} C$, for $j=1,2,3$, are algebraic integers in the number field $\mathbb{Q}\left(2 \cos (2 \pi / 5), e^{2 \pi i / 3}\right)$.

(iv) If $\tau=\sigma(\pi / 9,2 \pi / 7), \sigma(\pi / 9,4 \pi / 7)$ or $\sigma(\pi / 9,6 \pi / 7)$ then the entries of the matrices $\sqrt{3} C^{-1} H_{\tau} C$ and $e^{2 \pi i / 9} C^{-1} R_{j} C$, for $j=1,2,3$, are algebraic integers in $\mathbb{Q}\left(2 \cos (2 \pi / 7), e^{2 \pi i / 3}\right)$.

We then examine all Galois conjugations in $E$ that are compatible with nontrivial Galois conjugations in $F$. For the number fields in Lemma 6.7 the Galois conjugations permute the values of $\tau$ given in each part.

For example, in part (ii) of Lemma $6.7 E$ is $\mathbb{Q}(\sqrt{3}, \sqrt{5}, i)$ and $F$ is $\mathbb{Q}(\sqrt{3}, \sqrt{5})$. The Galois conjugation in $F$ fixing $\sqrt{3}$ and changing the sign of $\sqrt{5}$ extends to a Galois conjugation $g_{1}$ in $E$ that fixes $e^{2 \pi i / 3}$ and swaps $\sigma(\pi / 6, \pi / 5)$ and $\sigma(\pi / 6,2 \pi / 5)$ and swaps $\sigma(-\pi / 6, \pi / 5)$ and $\sigma(-\pi / 6,2 \pi / 5)$. Likewise, the Galois conjugation in $F$ fixing $\sqrt{5}$ and changing the sign of $\sqrt{3}$ extends to a Galois conjugation $g_{2}$ in $E$ that fixes $e^{2 \pi i / 3}$ and swaps $\sigma(\pi / 6, \pi / 5)$ and $\sigma(-\pi / 6+$ $2 \pi / 3, \pi / 5)$ and swaps $\sigma(\pi / 6,2 \pi / 5)$ and $\sigma(-\pi / 6+2 \pi / 3,2 \pi / 5)$. Note that $\sigma(\phi+$ $2 \pi / 3, \eta)$ is equivalent to $\sigma(\phi, \eta)$. The remaining Galois conjugation in $E$ is the product of $g_{1}$ and $g_{2}$.

In each case all nontrivial Galois conjugations will send our value of $\tau$ to one of the other sporadic values of $\tau$. Since we have analysed which of the points $\tau$ lie in our parameter space we know the signature of the corresponding Hermitian form $H_{\tau}$. In each case there will be at least one nontrivial Galois conjugation that sends $H_{\tau}$ to another form of signature $(2,1)$. This is sufficient to ensure that $\left\langle R_{1}, R_{2}, R_{3}\right\rangle$ is nonarithmetic; compare Corollary 12.2.8 of [Deligne and Mostow 1986]. This completes our sketch proof of Proposition 6.6.

\section{Acknowledgements}

This collaboration was made possible by the generous support of CNRS, Durham University, Université Pierre et Marie Curie (Paris 6), Johns Hopkins University, and the University of Utah.

\section{References}

[Conway and Jones 1976] J. H. Conway and A. J. Jones, "Trigonometric Diophantine equations (On vanishing sums of roots of unity)”, Acta Arith. 30:3 (1976), 229-240. MR 54 \#10141 Zbl 0349. 10014 
[Coxeter 1948] H. S. M. Coxeter, Regular polytopes, Methuen, London, 1948. MR 10,261e Zbl 0031.06502

[Deligne and Mostow 1986] P. Deligne and G. D. Mostow, "Monodromy of hypergeometric functions and nonlattice integral monodromy”, Inst. Hautes Études Sci. Publ. Math. 63 (1986), 5-89. MR 88a:22023a Zbl 0615.22008

[Deligne and Mostow 1993] P. Deligne and G. D. Mostow, Commensurabilities among lattices in PU $(1, n)$, Annals of Mathematics Studies 132, Princeton University Press, Princeton, NJ, 1993. MR 95a:22013 Zbl 0826.22011

[Goldman 1999] W. M. Goldman, Complex hyperbolic geometry, Oxford Mathematical Monographs, The Clarendon Press Oxford University Press, New York, 1999. Oxford Science Publications. MR 2000g:32029 Zbl 0939.32024

[Klimenko and Sakuma 1998] E. Klimenko and M. Sakuma, "Two-generator discrete subgroups of Isom $\left(\mathbf{H}^{2}\right)$ containing orientation-reversing elements", Geom. Dedicata 72:3 (1998), 247-282. MR 2000a:20111 Zbl 0928.20040

[Knapp 1968] A. W. Knapp, "Doubly generated Fuchsian groups", Michigan Math. J. 15 (1968), 289-304. MR 40 \#1483 Zbl 0167.07002

[Livné 1981] R. A. Livné, On certain covers of the universal elliptic curve, Ph.D. thesis, Harvard University, Cambridge, 1981.

[McReynolds 2006] D. B. McReynolds, "Arithmetic lattices in SU(n, 1)", preprint, 2006, Available at http://www.ma.utexas.edu/users/dmcreyn/ComplexArithmeticI.pdf.

[Mostow 1980] G. D. Mostow, "On a remarkable class of polyhedra in complex hyperbolic space", Pacific J. Math. 86:1 (1980), 171-276. MR 82a:22011 Zbl 0456.22012

[Mostow 1986] G. D. Mostow, "Generalized Picard lattices arising from half-integral conditions", Inst. Hautes Études Sci. Publ. Math. 63 (1986), 91-106. MR 88a:22023b Zbl 0615.22009

[Mostow 1988] G. D. Mostow, "On discontinuous action of monodromy groups on the complex n-ball”, J. Amer. Math. Soc. 1:3 (1988), 555-586. MR 89h:22020 Zbl 0657.22014

[Parker 2006] J. R. Parker, "Cone metrics on the sphere and Livné's lattices", Acta Math. 196:1 (2006), 1-64. MR 2007j:32021 Zbl 1100.57017

[Parker 2008] J. R. Parker, "Unfaithful complex hyperbolic triangle groups, I: Involutions", Pacific J. Math. 238 (2008), 145-169.

[Paupert 2005] J. Paupert, Configurations of lagrangians, fundamental domains and discrete subgroups of $\mathrm{PU}(2,1)$, Ph.D. thesis, University of Paris 6, Paris, 2005, Available at http://www.institut. math.jussieu.fr/theses/2005/paupert.

[Paupert 2007] J. Paupert, "Elliptic triangle groups in PU(2, 1), Lagrangian triples and momentum maps", Topology 46:2 (2007), 155-183. MR 2008f:53114 Zbl 1131.22010

[Pratoussevitch 2005] A. Pratoussevitch, "Traces in complex hyperbolic triangle groups", Geom. Dedicata 111 (2005), 159-185. MR 2006d:32036 Zbl 1115.32015

[Sauter 1990] J. K. Sauter, Jr., "Isomorphisms among monodromy groups and applications to lattices in PU(1, 2)”, Pacific J. Math. 146:2 (1990), 331-384. MR 92d:22016 Zbl 0759.22013

[Schwartz 2002] R. E. Schwartz, "Complex hyperbolic triangle groups", pp. 339-349 in Proceedings of the International Congress of Mathematicians (Beijing, 2002), vol. 2, edited by T. T. Li et al., Higher Ed. Press, Beijing, 2002. MR 2004b:57002 Zbl 1022.53034

Received November 28, 2007.

JOHN R. PARKER 
DePartment of Mathematical SCIENCES

DURHAM UNIVERSITY

SOUTH ROAD

DURHAM DH1 3LE

UNITED KINGDOM

j.r.parker@durham.ac.uk

http://www.maths.dur.ac.uk/ dma0jrp/

JULIEN PAUPERT

UNIVERSITY OF UTAH

DEPARTMENT OF MATHEMATICS

155 SOUTH 1400 EAST

SALT LAKE City, UT 84112

UNITED STATES

paupert@math.utah.edu

http://www.math.utah.edu/ paupert 